\title{
Chapter 17
}

X-ray Scattering Studies on Multiphasic Polymer Systems

17.1Introduction.

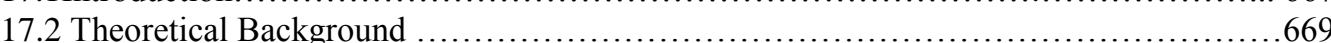

17.2.1 Microfibrillar Reinforced Composites (MCF): Definition and Preparation .............669

17.2.2 Clay-containing Polymer Nanocomposites.........................................6. 670

17.2.3 The use of WAXS and SAXS in Characterization of Polymers.........................671

17.3 Studies on Multiphase Polymer Systems .............................................6677

17.3.1 Polyamide 6/montmorillonite Nanocomposites..................................... 677

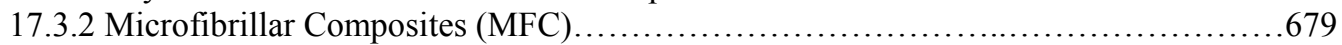

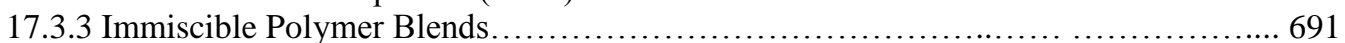

17.3.4 Non-conventional Molding of PP Nanocomposites.................................... 693

17.3.5 Stretching of Nanoclay PET Nanocomposite....................................... 695

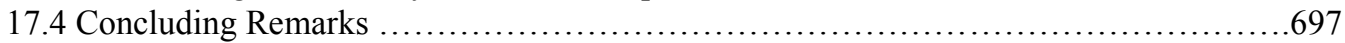

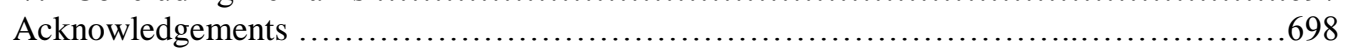

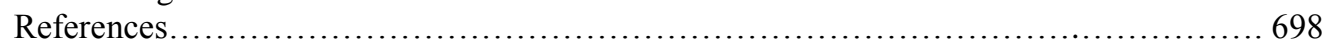




\title{
X-ray Scattering Studies on Multiphasic Polymer Systems
}

\author{
Z. Z. Denchev and J. C Viana \\ IPC - Institute for Polymers and Composites, University of Minho, Portugal
}

\subsection{Introduction}

At present, multiphase and multicomponent materials such as polymer alloys, blends, and composites consume over $80 \mathrm{wt} \%$ of all commercially-produced resins. The increase of this particular segment of the plastics industry is about three times faster than of the industry as a whole. The reason is that the modification by blending may improve significantly the resins' mechanical performance and processability, being at the same time cost effective. Those few resins that are used without adding of other components are most frequently semicrystalline, i.e. they already have a multiphase structure that makes other modification less urgent [1]. Recently, a large window has opened for new structural applications of the multicomponent polymer systems with the advent of nanoscale filled polymer composites. Changing the type, size, shape, volume fraction, interface, and degree of dispersion or aggregation of the nanofillers enables a great amount of unique combinations of properties with high potential for successful commercial development [2].

In technical literature the terms 'phase' and 'component' are often used interchangeably. In thermodynamics, however, a clear distinction is made. Thus, a phase is defined as a chemically and physically uniform quantity of matter that can be separated mechanically from a nonhomogeneous mixture. Hence, multiphasic polymer systems would comprise at least two different phases, e.g. semicrystalline single polymers featuring amorphous and crystalline phases or polymorphic polymers containing different concomitant crystalline phases. Many polymer systems with industrial importance such as blends, colloidal polymers, polymer composites/ filled polymers, etc. comprise two or more chemically distinct components, each one of them being able to contain various phases as well. These components may have different sizes, with microscopic to nanoscopic blocks being present.

Handbook of Multiphase Polymer Systems, First Edition; Eds.: Abderrahim Boudenne, Laurent Ibos, Yves Candau, and Sabu Thomas. ( 2011 John Wiley \& Sons, Ltd. Published 2011 by John Wiley \& Sons, Ltd. 
The wide use of multicomponent and multiphasic polymer systems in polymeric products fostered the investigations on their structure development during processing and the establishment of structures-properties relationships [3, 4]. Apart from their industrial importance, multiphase polymers and multicomponent materials on their basis are model systems in statistical physics for studying fundamental aspects of many properties such as conformational properties of the chains, the kinetics of phase transitions, as well as the detailed dynamics of diffusion processes [5]. The large molecular dimension of polymer systems markedly reduces the mixing entropy and provides the basis for self-organized structures [6]. Because of all these reasons, investigating polymer systems comprising many components and phases has become an important issue within modern materials science.

Generally, scattering methods are a useful tool to study a multiphase or multicomponent system since they are sensitive to the spatial inhomogeneities due to composition or phase fluctuations in polymer materials, either amorphous or semicrystalline. Many relevant studies in this field have been performed by means of small-angle scattering of X-rays (SAXS) or of neutrons (SANS) [7]. The latter technique is less accessible due to the necessity of nuclear reactors and special safety precautions. The wide angle scattering of X rays (WAXS) called also $\mathrm{X}$-ray diffraction is very frequently used in characterization of semicrystalline multiphase systems. The diffraction pattern contains information that is specific to each phase within the illuminated volume, including both geometric and structural parameters, many of which are inaccessible to other techniques. It is a common feature of all scattering techniques that the structural information can be collected non-invasively, providing in situ and real-time possibilities. While these capabilities already turn WAXS and SAXS into quite powerful techniques, the output can be considerably enhanced by collecting data in synchrotron beamlines, using high-flux and, whenever possible, micro-focused X-ray beams [8].

Although synchrotron is a good option for doing WAXS and especially SAXS, some benchtop equipment is also available (e.g. with a Kratky camera or with a two-dimensional detector as the NanoStar analyzer of Bruker AXS) capable of yielding good SAXS data. Characterization of multicomponent/multiphase polymer systems by X-ray techniques is a vast scientific area. It includes by definition the studies on all polymer blends, alloys and composites that are made of chemically-independent constituents (components). Moreover, each semicrystalline polymer is to be considered at least biphasic, comprising a disordered (liquid) amorphous fraction and a solid fraction with crystalline order. If the latter is made of various crystalline phases (polymorphs) even a semicrystalline homopolymer can be considered a multiphase system.

With all these ideas in mind, the scope of the present chapter had to be limited to some recent studies on the application of synchrotron WAXS and SAXS in three particular multicomponent and multiphase polymer systems. The first system comprises materials that became known as microfibrillar reinforced composites (MFC) produced from oriented blends of thermoplastic semicrystalline polymers by conventional processing techniques. These materials belong to fiber-reinforced composites that have many important engineering applications but are notoriously difficult to study [9].

As a second material system, the structure development during processing of an immiscible polymer blend of polypropylene (PP) and polystyrene (PS) was investigated by Xray scattering techniques. Structure formation in polymers blends has been widely investigated in the last years, mainly in terms of the development of the size, shape, and orientation of the dispersed component under flow deformation [10]. Further, the structure evolution and damage during stretching in the solid state of polymers blends is much less researched topic. Complementing, this second study, the structure evolution of the PP/PS blend was investigated by time resolved x-ray scattering in a synchrotron source.

Finally, the third case reveals investigations on the structure of polymer nanocomposites developed during processing and also during stretching. Polymer nanocomposites are a recent class of materials, and very few studies have been published on the structure development in them (e.g. [11, 12]). 
In this chapter, it will be also demonstrated how WAXS and SAXS can be used, along with other characterization techniques, to characterize the structure of multicomponent and multiphase polymer systems and how their nanostructure relates to their mechanical behavior and properties.

\subsection{Theoretical Background}

\subsubsection{Microfibrillar Reinforced Composites (MCF): Definition and Preparation}

The MFCs, described initially about two decades ago [13-15], are a special type of in situ nanocomposites combining the easier preparation and processability of the conventional polymer fibrous composites with the presence of micro- and nanosized, high aspect ratio reinforcements typical of the nano- and molecular composites. In the MFCs these reinforcements are fibrils built of bundles of flexible, organic macromolecules produced by appropriate mechanical and thermal treatment of a polymer blend. The typical diameters of the reinforcing fibrils in MFC were found to be within the upper size limit of nanocomposites (i.e. $100-1000 \mathrm{~nm}$ ). Nevertheless, they could hardly be considered typical representatives of either macro- or nanocomposites [16].

The preparation of MFCs is quite different from that of the conventional composites, insofar as the reinforcingmicro- or nanofibrils are created in situ during processing, as is the relaxed, isotropic thermoplastic matrix. The preparation of MFCs comprises three basic steps $[17,18]$. First, melt-blending is performed of two or more immiscible polymers with melting temperatures $\left(T_{m}\right)$ differing by at least $30^{\circ} \mathrm{C}$. In the polymer blend so formed, the minor component should always originate from the higher-melting material and the major one from the lower melting component or could even be amorphous. Second, the polymer blend is drawn at temperatures equal or slightly above the glass transition temperatures $\left(T_{g}\right)$ of both components leading to their orientation (i.e. fibrillation). Finally, liquefaction of the lower melting component is induced thus causing a nearly complete loss of orientation of the major phase upon its solidification, which, in fact, constitutes the creation of the composite matrix. This stage is called isotropization. It is very important that during isotropization the temperature should be kept below $T_{m}$ of the higher melting and already fibrillated component. In doing so, the oriented crystalline structure of the latter is preserved, thus forming the reinforcing elements of the MFC.

Although MFCs are based on polymer blends, they should not be considered oriented blends. It is the stage of isotropization where the latter are transformed into composite materials. Along with the loss of orientation of the matrix, depending on the chemical functionality of both reinforced and reinforcing components, chemical reactions may also take place resulting in the formation of a copolymeric interface. This interface plays the role of a compatibilizer increasing the adhesion between the matrix and the reinforcing components. If no chemical functionality is present, suppressing of incompatibility between the two materials may be achieved by adding compatibilizing agents to strengthen the interface.

In the first studies on MFCs, the composites were prepared on a laboratory scale performing every one of the aforementioned three processing stages separately, one after another. Blending was realized in a laboratory mixer or a single-screw extruder to obtain nonoriented strands that were afterward cold-drawn in a machine for tensile testing, followed by annealing of the oriented strands with fixed ends [13-15, 19-22]. Obviously, this discontinuous scheme is difficult to apply in large-scale production. More relevant in this case are the continuous setups developed more recently [23-26]. Blending of the components and extruding the initial strands could be performed in a twin-screw extruder coupled with two or more drawing devices [24].

After the extrusion blending-drawing stage, one obtains the polymer blend at the exit of the second haul-off device in the form of oriented, continuous cables (OC). To perform the matrix isotropization stage, these strands are further processed by compression molding at temperatures above $T m$ of the matrix and below $T m$ of the reinforcing fibrils, whereby the 
former melts assuming the form of the mold and embedding the bundles of oriented fibrils whose orientation and length may be varied [27].

Other molding techniques can also be used instead of compression molding. Monticciolo et al. [28] and later on Pesneau et al. [29] and Evstatiev et al. [30] used an approach in which after the fibrillation of the respective blend by drawing, the oriented strands were cooled down to freeze the morphology and chopped to pellets. The latter were reprocessed by second extrusion or by injection molding at a temperature below the $T m$ of the dispersed fibrillated component.

In this chapter an attempt is made to explain the mechanical properties of polyamide reinforced MFCs with a HDPE matrix relating them to their structure using synchrotron WAXS and SAXS complemented by scanning electron microscopy (SEM).

\subsubsection{Clay-containing Polymer Nanocomposites}

Polymer nanocomposites exhibit substantial improvement of their properties as compared with those of the virgin raw materials and their traditional microcomposites [11, 12, 31, 32]. From those, nanoclay-reinforced polymers received a lot of industrial and scientific interest as they show improved mechanical properties, higher thermal resistance, reduced gas permeability and reduced flammability $[11,32]$. The influence of the incorporation of nanoclays on the structure and hierarchical organization of a polymer, and therefore on their properties, has been investigated. When fillers of size comparable with the segmental blocks of macromolecules are added to the polymeric systems they interfere at the molecular level giving unusual properties, at low levels of incorporation (even less than 1\%) [31,33]. For nanoclay-filled polymers, structural features as nanoparticles dispersion and exfoliation level influence the nanocomposite properties $[34,35]$. When adding a nanoclay to a polymer three materials may be obtained depending upon the degree of separation of nanoclay layers (Figure 17.1): (i) a fully exfoliated clay, resulting in a true polymer nanocomposite; (ii) intercalated clays with increased intergallery distance due to the insertion of the polymer macromolecules;
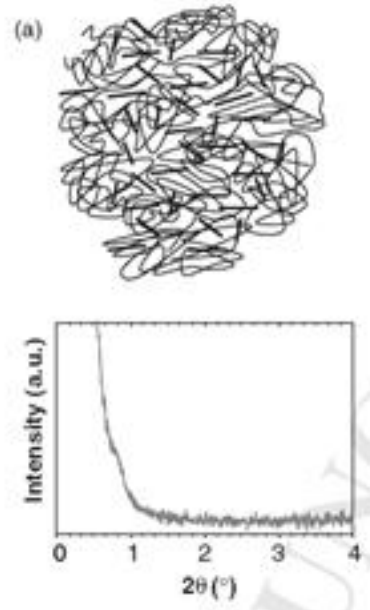

(b)
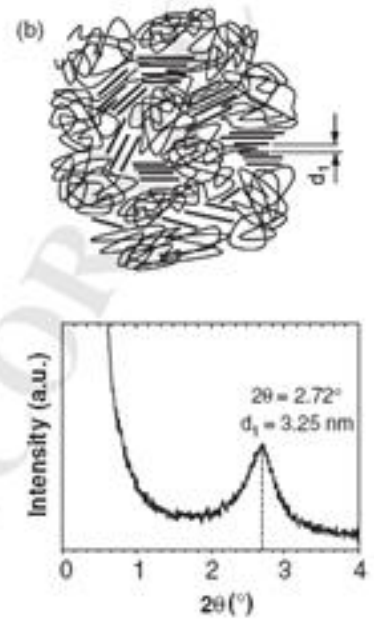
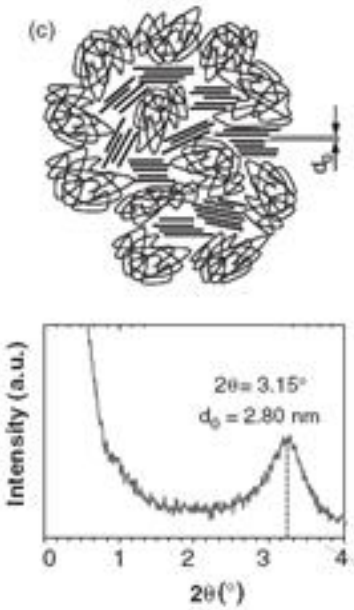

Figure 17.1 Structure of a clay-filled polymer and respective Intensity-20 plot (WAXS): (a) exfoliated clay nanocomposite; $(b)$ intercalated composite $\left(d_{1}-\right.$ clay inter-gallery distance; $\left.d_{l}>d_{0}\right)$; $(c)$ agglomerated (tactoids) microcomposites ( $d_{0}$ - pristine clay inter-gallery distance). 
and (iii) agglomerated nanoclays, resulting in a traditional polymer microcomposite. In most of the cases, fully exfoliation of the nanoclays is difficult to achieve and the property enhancement is reduced.

Due their nanometer size, X-ray diffraction (WAXS) is able to measure the clay's intergallery distance. However, for very low levels of incorporation of nanoclays, the absence of a reflection peak is not a direct evidence of their fully exfoliation. The agglomeration/exfoliation and dispersion of the nanometric reinforcements in the polymeric matrix is essential for improved mechanical behavior [32]. Contrasting with the innumerous studies on polymer nanocomposites in the last years, the evolution of the structure during deformation has been investigated scarcely [36].

In this chapter, the structure development during processing of nanoclay-filled PP is investigated. The morphology of PP is characterized for nanocomposites with different amounts of incorporation of nanoclays. Furthermore, the structure evolution during stretching of PET nanocomposites is studied by in situ SAXS investigations. This aims at giving fundamental insights about deformation mechanisms at the nanoscale with adequate time-resolution.

\subsubsection{The use of WAXS and SAXS in Characterization of Polymers}

$\mathrm{X}$-rays are electromagnetic radiation occupying the spectrum from $10^{-2}$ to $10^{2} \AA$ in wavelength. Scattering experiments with polymers are performed mostly with the $\mathrm{K} \alpha$ characteristic radiation from a copper target tube with $\lambda=1.5418 \AA$. X-rays of similar wavelength can also be selected, by means of monochromator, from the broad spectrum emitted by a synchrotron radiation source.

The setup scheme of a scattering experiment in symmetrical-transmission mode frequently used in synchrotrons is represented in Figure 17.2(a). There, radiation from the storage ring source (1) is monocromatized by the incident beam optics (2). The sample (3) is in upright position and the primary (unscattered) beam passes through it hitting the beamstop (5). The detector (4) collects only the X-rays scattered at various $2 \theta$ angles. This setup allows the easy change of the sample-to-detector distance $R$, but requires a two-dimensional detector for recording the complete scattering pattern.

Figure 17.2(b) shows a scheme of the classical symmetrical-reflection geometry X-ray setup. With this setup the angle $2 \theta$ is changed while recording the intensity of the scattered radiation measured typically by a linear detector. To obtain the complete scattering pattern, the sample should be tilted and/or rotated.

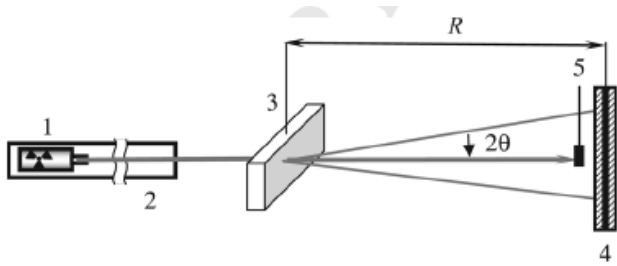

(a)

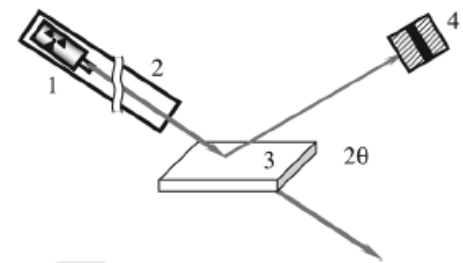

(b)

Figure 17.2 (a) - Symmetrical-transmission geometry of the X-ray setup; (b) - Symmetrical-reflection geometry $X$-ray setup. 1 -X-ray source; 2 - Incident beam optics; 3 - sample; 4 -detector; 5 - beamstop; $R$ - sample-to-detector distance. 
Table 17.1 Subareas of scattering as a function of the sample-to-detector distance $R$ assuming an X-ray wavelength of $\lambda \approx 0.15 \mathrm{~nm}$. [8].

\begin{tabular}{lll}
\hline Subarea & $R, \mathrm{~m}$ & Focus \\
\hline WAXS & $0.05-0.2$ & Arrangements of chain segments \\
MAXS & $0.2-1.0$ & Liquid-crystalline structure \\
SAXS & $1.0-3.0$ & Nanostructure $3-50 \mathrm{~nm}$ \\
USAXS & $6.0-15.0$ & Nano and microstructure $15 \mathrm{~nm}-2000 \mathrm{~nm}$ \\
\hline
\end{tabular}

Scattering experiments in multiphase polymer systems are carried out in four different angular regions (subareas) shown in Table 17.1 [8]. When the symmetrical-transmission geometry is used, switching between the various subareas is made by an arbitrary change of the distance $R$.

The scattering patterns obtained in the WAXS subarea yield information on the arrangement of polymer chain segments e.g., orientation of the crystalline and amorphous phases, crystalline structure, size of crystals, crystal distortions, WAXS crystallinity. The subarea of middle-angle X-ray scattering (MAXS) covers the characteristic scattering of liquidcrystalline structure and rigid-rod polymers. In the SAXS regime the typical nanostructures are observed. Because of the long distance between sample and detector time-resolved measurements can only be carried out at synchrotron radiation sources. The ultra small-angle Xray scattering (USAXS) extends the accessible structure towards the micrometer range. Timeresolved measurements require a synchrotron beam that is intensified by an insertion device [8]. The most frequently used subareas in scattering experiments with polymers are WAXS and SAXS.

Generally, the interactions of X-rays with matter produces two different phenomena [7]: (1) scattering of $\mathrm{x}$-rays by the individual electrons in the sample, and (2) interference among the various scattered waves. Clearly then, the term scattering refers only to phenomenon 1, while the term diffraction is related to the combination of 1 and 2. In fact, this distinction is often omitted. Thus, when the scattering pattern is diffuse and especially if it is in the SAXS subarea, the term scattering is exclusively used, even if some interference is involved. The term diffraction tends to be used when the sample is crystalline, i.e. sufficiently regular so as to concentrate the scattered beam around a number of sharply defined scattering directions, as is in the WAXS subarea of semicrystalline polymers.

In principle, the X-ray experiment measures the X-ray flux (i.e. the intensity of the scattered radiation, $I s$ ), as a function of the scattering direction, determined by the scattering vector $s$ or scattering angle, $2 \theta$ (Figure 17.3). These data are then analyzed and interpreted so as to obtain information about the relative placements of electrons in the sample. The theory of the $\mathrm{X}$-ray scattering in polymers has been rigorously developed and extensively described in the specialized literature [7, 8, 37, 38].

This chapter will only focus on some specific applications of WAXS and SAXS useful for characterization of multiphase and multicomponent systems with the emphasis on the applied aspects and not on the theoretical and mathematical ones.

\subsubsection{Degree of Crystallinity by WAXS}

The scattering from an amorphous material such as a melt or a glass gives an intensity pattern which is broad and essentially featureless except for the so-called amorphous halo. On the other hand, the diffraction pattern obtained from a crystalline material consists of series of sharp Bragg peaks, easily distinguishable from the diffuse background (Figure 17.2). Consequently, a semicrystalline polymeric material will give a pattern representing the superposition of both of these features, whereby their relative contributions will reflect the relative amounts of the non- 

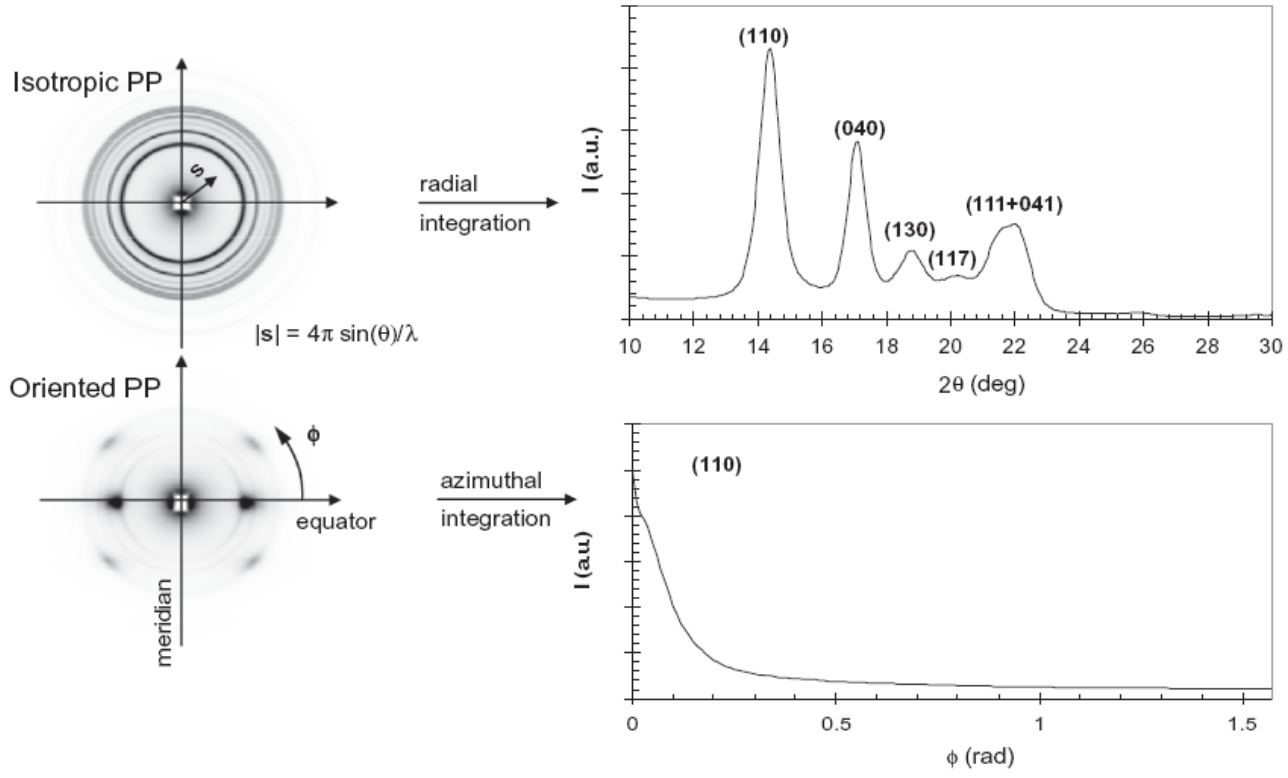

Figure 17.3 2D WAXS patterns of an isotropic and highly oriented (stretched) PP samples and the correspondent radial and azimuthal integration profiles (orientation direction is vertical).

crystalline and crystalline phases present. That is why, to turn diffraction measurements into a quantitative tool for evaluating the degree of crystallinity, it is necessary to separate the observed intensity into crystalline and non-crystalline components. After this is done, the degree of crystallinity $X c$ can be defined as [6]:

$$
X_{C}=\frac{Q_{c r}}{Q}=\frac{4 \pi \int_{0}^{\infty} s^{2} I_{c r}(s) d s}{4 \pi \int_{0}^{\infty} s^{2} I(s) d s}
$$

where $s$ is the scattering vector, $I(s)$ is the total scattered intensity, $I_{c r}(s)$ is its crystalline component and $Q$ and $Q_{c r}$ are the corresponding scattering invariants.

There are several limitations for the practical application of the above relation. First, it will hold strictly only for non-oriented (isotropic) samples. For anisotropic materials the wideangle scattering must be recorded as a function of both scattering angle and sample orientation in a texture goniometer [8], before the data can be isotropized and the exact values for $Q$ and $Q_{c r}$ can be calculated. Second, experimentally, the intensities $I(s)$ and $I_{c r}(s)$ are available only up to a finite upper limit of $s$ and not to infinity, i.e, there will always be a systematic error in Xc due to the truncation of both integrals. Moreover, the lattice imperfections in the crystalline phase cause that part of the scattering intensity of the Bragg peaks to be diverted to the amorphous halo leading to underestimation of $X c$. The problems caused by the data truncation and lattice imperfections are resolved by the method for $X c$ determination proposed by Ruland [39]. 
where FWHMh,k,l is the full width at half maximum of (h,k,l) peak, $\lambda$ is the X-ray wavelength

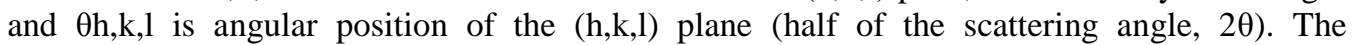
determination of FHMHh,k,l must be performed in peaks that do not overlap or after the peak's deconvolution. However, this latter option may induce relatively high errors in the estimation of crystal size. Moreover, the peak width can be broadened by a distribution of Dhkl on the sample or straining of the crystal lattice, leading to a sub-estimation of the crystal size.

\subsubsection{Phase Orientation Studies by WAXS}

The orientation of the $(\mathrm{h}, \mathrm{k}, \mathrm{l})$ reflection planes can be assessed by WAXS. In an oriented semicrystalline polymer, the intensity of each reflection is very dependent upon the azimuthal angle $(\varphi)$. Assuming a given unit crystal geometry, the intra-chain orientation $(0,0,1)$ is revealed on the meridian and the inter-chain $(\mathrm{h}, \mathrm{k}, 0)$ on the equator. The more concentrated is the intensity in a reflection arc the higher is the level of crystalline phase orientation. This level of orientation is calculated from azimuthal profiles (between azimuthal angle $\varphi=0 \div \pi / 2$ rad or $\varphi=0 \div \pi$; Figure 17.3). The crystalline phase orientation is normally assessed by the average square of the cosine of $\varphi$ (the angle between the crystallographic plane and the reference direction), $\langle\cos 2 \phi\rangle$, defined as (assuming an uniaxial symmetry): 
where, $I(\varphi)$ is the scattered intensity of $(\mathrm{h}, \mathrm{k}, \mathrm{l})$ plane as function of $\varphi$. The crystalline phase orientation is normally expressed by the Hermans' orientation function, $f$, (or second moment of orientation function) defined by:

$$
f=\frac{3\left\langle\cos ^{2} \phi\right\rangle-1}{2}
$$

According to Eq. (17.5), if all chains are oriented on the director direction $\mathrm{f}=1$; for fully transverse orientation $f=-0.5$; and $f=0$ for random orientation. If the plane normal coincides with a crystallographic axis $(\mathrm{a}, \mathrm{b}$ and $\mathrm{c})$ and for orthorhombic unit cell geometry, the orientation of the different unit crystal planes ((100), (010) and (001)) is interrelated by:

$$
\left\langle\cos ^{2} \phi_{100}\right\rangle+\left\langle\cos ^{2} \phi_{010}\right\rangle+\left\langle\cos ^{2} \phi_{001}\right\rangle=1
$$

More generally, if the crystallographic planes are not parallel and other planes of orientation are known, the other $\mathrm{f}$ can be calculated by using the Wilchinsky's method [40].

\subsubsection{Structure Investigations by SAXS}

As seen from Table 17.1, SAXS is used to study structures of size on the order of $3 \mathrm{~nm}$ and larger. Information on such relatively large structures can be collected at scattering angles $2 \theta$ lower than $2^{\circ}$. The relation between the dimensions in the real space and those in the reciprocal space is given by the Bragg law:

$$
d=\frac{\lambda}{2 \sin \theta}=s^{-1}
$$

Hence, if the distance $d$ representing the period of repetition in the structure, (e.g. the distance between the similar domains), is around $10 \mathrm{~nm}$ and larger, the corresponding scattering angle $2 \theta$ will be about $0.6^{\circ}$ or smaller, i.e. the observations will be made in the SAXS subarea. On the other hand, the typical distances between the crystallographic planes in polymers are in the order of few Angstroms, therefore the scattering angle will be typically about $20^{\circ}$, i.e. in the WAXS subarea.

It should be noted that all methods developed for the analysis of WAXS data are applicable in SAXS analysis as well. In the latter case there exist theoretical results dedicated specifically for SAXS data treatment. For example, in small angle scattering $\sin \theta$ can always be approximated by $\theta$. Moreover, in the SAXS analysis it is assumed that any details of size scale less than $0.1 \mathrm{~nm}$ do not exist. In the simplest and most frequently-used analysis of SAXS data, the observed peak of the scattering curve is related to the average distance between the nanoscopic domains in the sample, called also the long period, $L$. Hence, for small scattering angles and based on the reciprocity in the Bragg law:

$$
L=1 / s_{\max }
$$

In Eq. (17.8) $L$ represents the sum of the average thickness of the crystal lamellae, $l_{c}$ and of the interlamellar amorphous regions, $l_{a}$.

In isotropic and moderately oriented polymer samples $s_{\max }$ must be measured only after background subtraction and Lorentz correction of the curve [8], i.e. after changing the y-axis from $I(s)$ to $s^{2} . I(s)$. Only in this case the $L$-data obtained directly from the scattering curve maxima are close to the correct ones calculated by more rigorous methods [41]. Highly aniso- 
tropic materials that show intensive peaks on a relatively low background do not require Lorentz correction. In this chapter, the correct $s_{\max }$ values of various multiphase polymer systems were obtained by the above procedure and discussed as a function of the materials processing parameters.

Apparently, Eq. (17.8) cannot be used for determination of $l_{c}$ and $l_{a}$. To do that, the approach of Kortleve and Vonk [42] elaborated for the case of isotropic polymers is to be employed. The Fourier transform of the Lorentz corrected SAXS profile is calculated, namely the linear correlation function $\gamma_{1, r}(\mathrm{CF})$ as:

$$
\frac{\gamma_{1, r}}{Q}=\frac{\int_{0}^{\infty}\left(I-I_{b}\right) q^{2} \cos (q r) \exp \left(\sigma^{2} q^{2}\right) \mathrm{d} q}{\int_{0}^{\infty}\left(I-I_{b}\right) q^{2} \mathrm{~d} q}
$$

Here, $q=2 \pi . s ; I_{b}$ is that contribution to the total scattering arising from density fluctuations (liquid scattering), and $\sigma$ is a term, related to the thickness of the crystal/amorphous interface. $Q$ is the so-called scattering invariant that can be determined by integrating the SAXS profile over all scattering angles, i.e.:

$$
Q=\int_{0}^{\infty}\left(I-I_{b}\right) q^{2} \mathrm{~d} q
$$

The advantage of the CF method in contrast to Bragg's law is that, in addition to the long period $L$ the values for $l_{c}$ and $l_{a}$ and the degree of crystallinity within the lamellar stacks $\left(x_{c l}\right)$ (also called linear crystallinity) can be obtained.

In addition to the $L$ value, the $\mathrm{CF}$ approach calculates for each sample two additional estimates for the long spacing - from the position of the first maximum of CF (denoted as $L_{c}^{M}$ ) and from twice the position of the first minimum of CF $L_{c}^{m}$ ). To calculate the values of $l_{a}$ and $l_{c}$ on the basis of $\mathrm{CF}$, the following equation was used:

$$
\frac{B}{L_{c}^{M}}=x_{1}\left(1-x_{1}\right)
$$

where $\mathrm{B}$ is the position of the first intercept of CF with the $r$-axis. From the two solutions $x_{1,2}$ of the above quadratic equation, the one with the higher value is ascribed to the larger fraction of the two phases found within the lamellar stacks. For example, in highly crystalline samples, $x_{2}$ would correspond to the crystal fraction within the lamellar stacks (denoted as $x_{c l}$ ) and $1-x_{c l}$ would, then, represent the amorphous fraction within the stack.

Once the assignment of $x_{c l}$ is made for each particular case, one may calculate the $l_{c}$ and $l_{a}$ from the values of $L$ employing the following equations:

$$
l_{c}=x_{c l} L \quad \text { and } \quad l_{a}=\left(1-x_{c l}\right) L .
$$

where $L$, as indicated in [5], may take the values of $L_{c}^{M}$, or $L_{c}^{m}$

The $\mathrm{CF}$ analysis [42] and the more recently developed linear interface distribution function (IDF) [43] are not applicable in anisotropic polymer systems such as MFCs, but work well in clay-filled polymers. Some recent examples of the application of the CF approach in PA6/montmorillonite nanocomposites will be given in Section 17.3.1. Other applications of CF and IDF approaches in isotropic multiphase polymers can be found in [44-46]. 


\subsection{Studies on Multiphase Polymer Systems}

\subsubsection{Polyamide 6/montmorillonite Nanocomposites}

It is important to note that in these two-component systems the SAXS patterns can be treated quantitatively to extract two types of structural information: related to the polymer matrix and related to the inorganic filler. Here, the study of the polymeric part of the composite will be exemplified. Some recent studies on the SAXS patterns due to clay are also available [47-49].

\subsubsection{Materials and Sample Preparation}

This series was prepared by extrusion-blending of neat PA6 with a PA6/nanoclay masterbatch containing 20\% of montmorillonite (MMT) supplied by Nanocor. After compression molding of the respective granulates, isotropic PA6 nanocomposites in the form of laminate plates were obtained in which the MMT content varied between 1 and $7.5 \mathrm{wt} . \%$.

\subsubsection{Experimental Techniques}

The structure of the PA6/MMT laminates was studied by SAXS in the setup previously described. The 2D SAXS patterns were cut to obtain the respective 1D scattering profiles which were then analyzed by the SASDAP software (Copyright $(1995$ by R. Verma, A. Biswas and B. Hsiao, DuPont Experimental Station, Wilmington, DE, USA). This software computes the linear CF and derives values for $L_{c}^{M}, L_{c}^{m}, l_{a}$ and $l_{c}$ according to Eqs (17.8-17.12).

\subsubsection{Structure of PA6/MMT Composites}

Figure 17.4 shows the dependence of the Bragg long spacing $L B$ on the MMT content. It can be seen that with the increase of the MMT content, the intensity of the PA6 peak decreases until it completely disappears in the PA6/5\% MMT sample.

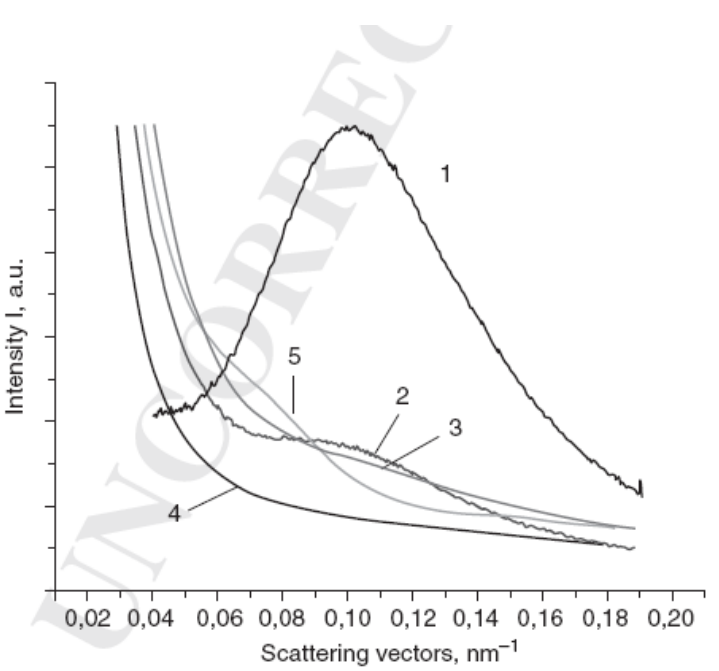

Figure 17.4 ID SAXS profiles of PA6/MMT nanocomposites containing various amounts of montmorillonite clay, wt.\%: 1 - no MMT, 2-1\%;3-2.5\%; 4-5\%; 5-20\% (Nanocor masterbatch). 
Table 17.2 Comparison of structural parameters obtained by WAXS and SAXS as a function of the MMT content in PA6/MMT nanocomposites. For designations see the text.

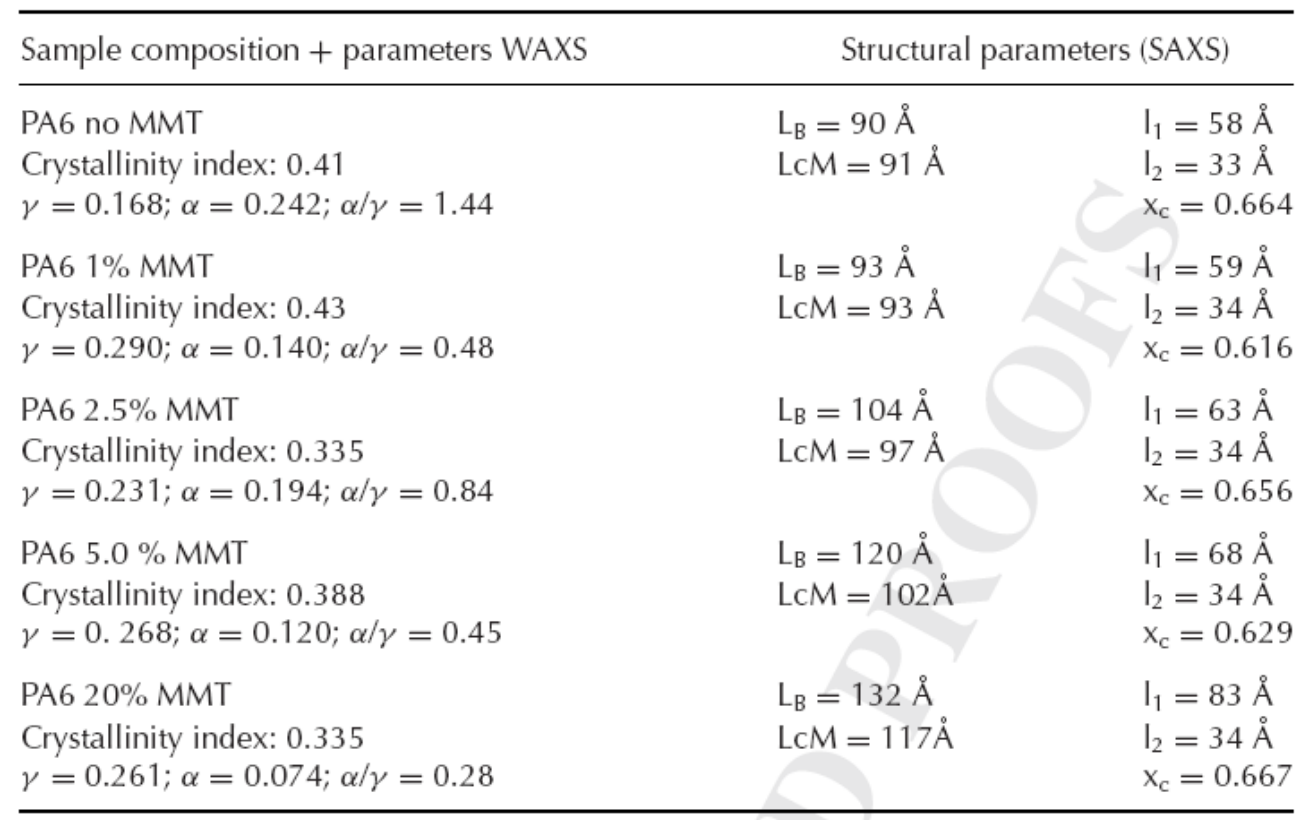

It seems that the introduction of MMT decreases the density difference between the amorphous and crystalline PA6 phases. In the sample with the largest amount of MMT, it is the amorphous phase that becomes denser. The reappearance of the density difference results in a new SAXS peak corresponding to larger $L_{B}$ (Table 17.2). As a result of this observation one may conclude that, if a simple two-phase model is considered for the PA6 matrix, the nanoclay is distributed within the amorphous phase and not within the crystalline one.

Figure 17.5 shows the CF curves of the PA6/MMT composites compared to that of the neat PA6 matrix. Curves 4 and 5 reveal larger long spacing values (denoted in Table 17.2 with $L_{c}^{M}$ to distinguish from those obtained from the raw SAXS profiles), being with broader size distributions, as compared to the CFs of samples 1-3.

The CF analysis allows the division of $L_{c}^{M}$ into two parts: $l_{l}$ (larger) and $l_{2}$ (smaller) values, corresponding to either the average thickness of the crystal lamellae, $l_{c}$, or to that of the interlamellar amorphous layers, $l_{a}$. Table 17.2 contains also the values of the intra-stack crystallinity fraction denoted as $\mathrm{x}_{\mathrm{c}}$. As explained in the notes to Eq. (17.11) above, the value of $\mathrm{x}_{\mathrm{c}}$ is supposed to be always higher than the average crystallinity index, obtained byWAXS. The assignment of the $l_{1}$ and $l_{2}$ values is only possible after consideration of all data from WAXS and SAXS in the table. Thus, with the increase of the MMT content there is a general trend toward a slight diminution of the average WAXS crystallinity index, whereby the fraction of the $\gamma$-PA6 polymorph notably increases. At the same time, both $L B$ and LcM values increase, due to the augmentation of the larger size $l_{1}$, whereas the $l_{2}$ values remain constant. Having in mind the explanation of the intensity changes of the SAXS peak in Figure 17.4, it can be concluded that the modification of PA6 with MMT results in the expansion of the amorphous phase, in which the MMT is concentrated, i.e. $l_{l}=l_{a}$ and $l_{2}=l_{c}$. Forthcoming studies in this PA6/MMT system will allow correlating the said nanostructural changes with the mechanical properties of the respective nanocomposites. 
Figure 17.5 Linear correlation function curves of PA6/MMT nanocomposites containing various amounts of montmorillonite clay, wt. \%: 1 - no MMT, $2-1 \% ; 3-2.5 \% ; 4-5 \% ; 5-20 \%$ (Nanocor masterbatch).

\subsubsection{Microfibrillar Composites (MFC)}

\subsubsection{Materials and Sample Preparation}

For the preparation of the oriented MFC precursors, the selected amounts of the HDPE, PA6 and YP granulates were premixed in a tumbler in various proportions. Each mixture was introduced into a K-Tron Soder gravimetric feeder that fed it to the hopper of a laboratory modular Leistritz LSM 30.34 intermeshing co-rotating twin-screw extruder [50].

At the exit of the last haul-off device the blends are in the form of oriented, continuous cables (OCs). The OCs were cut and aligned in the form of unidirectionally arranged bundles (unidirectional ply laminate, UDP. This was subjected to selective melting whereby isotropization and controlled crystallization of the matrix occurred in a hot press at a fixed temperature of $160^{\circ} \mathrm{C}$, a pressure of $2 \mathrm{MPa}$ and a cooling rate of ca. $10^{\circ} \mathrm{C} / \mathrm{min}$. Standard rectangular laminate plates $(60 \times 120 \mathrm{mmwith}$ a thickness of $0.1-1.5 \mathrm{~mm})$ were obtained from all the precursors. They were used for structural and morphological characterization, as well as to yield specimens for the tensile tests.

\subsubsection{Experimental Techniques}

Scanning ElectronMicroscopy (SEM) To analyze the morphology of theMFCs and their precursors, SEM of freeze-fractured specimens was used. For each blend, specimens were collected for morphological analysis typically at three different locations of the extruder line: at the extrusion die, after the first and after the second haul-off units. The final MFCs obtained after compression molding were also analyzed. All samples were sputter-coated with gold and observed in a Leica S360 SEM at magnifications of $\times 2.0 \mathrm{k}, \times 5.0 \mathrm{k}$ and $\times 7.5 \mathrm{k}$. Most of the specimens studied were obtained by cryogenic fracture with liquid nitrogen and the fractured surfaces were observed by SEM. 
$X$-ray Measurements Details All WAXS and SAXS patterns in this study were registered at the Soft Condensed Matter Beamline (A2) of HASYLAB, Hamburg, Germany using synchrotron radiation with a wavelength fixed to $0.15 \mathrm{~nm}$, using the symmetrical transmission geometry (Figure 17.2(a)). The sample-to-detector distance for SAXS was set at $2830 \mathrm{~mm}$, the diffraction patterns being registered by means of a MAR CCD 2D detector with exposure times of $30 \mathrm{~s}$. For the WAXS measurements the detector was positioned at $90 \mathrm{~mm}$ in respect to the sample. The various MFCs were studied in transmission mode, the sample thickness being in the $0.1-$ $1.5 \mathrm{~mm}$ range. Scattering patterns were obtained at certain temperatures in the $30-300^{\circ} \mathrm{C}$ range employing a typical heating rate of $20^{\circ} \mathrm{C} / \mathrm{min}$. A specially designed sample holder was used allowing for a controlled heating/cooling of the sample in the $25-300^{\circ} \mathrm{C}$ range. An IMAGO multi-channel process and program controller of JUMO GmbH \& Co. KG was used to regulate the sample temperature in heating or cooling at various rates. The difference between the readout and real temperature of the sample was found to be $3-4{ }^{\circ} \mathrm{C}$ at a heating or cooling rate of $20^{\circ} \mathrm{C} / \mathrm{min}$

\subsubsection{Results and Discussion}

SEM of HDPE/PA6/YP MFC The SEM images of the final MFCs (Figure 17.6, 1-4b) confirm that: (i) the PA6 reinforcing component has well-expressed fibrillar morphology, and (ii) the average diameter of these fibrils is in the upper nanometer - lower micrometer range, e.g. from 0.6 to $1.5 \mu \mathrm{m}$ (samples without compatibilizer) and from 0.5 to $1.0 \mu \mathrm{m}$ (compatibilized samp-

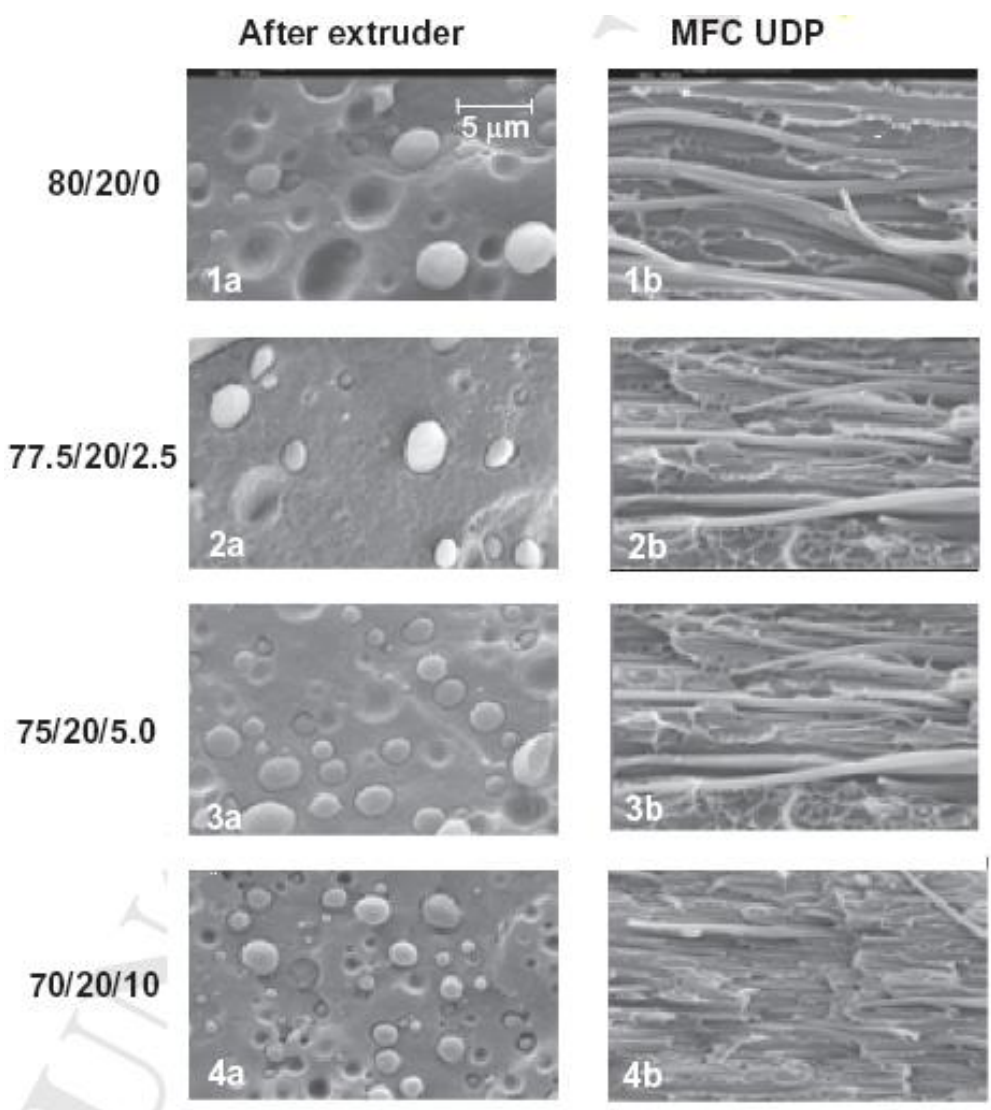

Figure 17.6 SEM images of various HDPE/PA6/YP materials after cryogenic fracture at the various stages of the MFCs preparation (compositions given in wt.\%): non-oriented blend right after the extruder die (1-4 (a)); UDP composites fractured along the fibrils axis (1-4 (b)). 

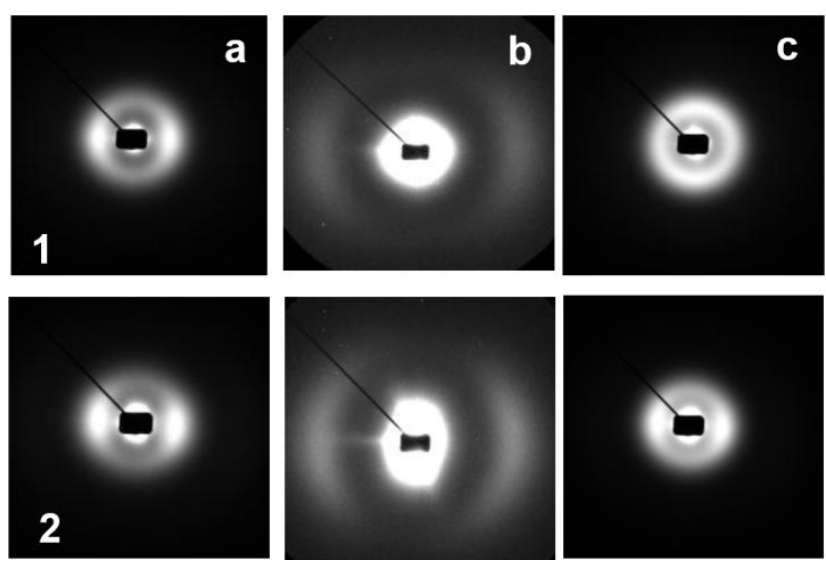

Figure 17.7 2D SAXS images of two HDPE/PA6/YP UDP MFC with compositions: 1 - 80/20/0; 2 70/20/10; at different temperatures: (a) - pattern of starting $\mathrm{MFC}$ at $30{ }^{\circ} \mathrm{C} ;(\mathrm{b})-$ pattern at $160^{\circ} \mathrm{C}$, heated in the beam; (c) - pattern at $30^{\circ} \mathrm{C}$ after heating at $160{ }^{\circ} \mathrm{C}$. The fibril axis is horizontal [47].

les). Therefore, depending on the compatibilizer content, the resulting composites can be considered as either nanostructured (NPC) or microfibrillar (MFC). Further on the second abbreviation the second abbreviation will be accepted.

None of the images of MFCs in Figure 17.6 permits measuring directly the fibrils' lengths. Their average lengths and aspect ratios of the reinforcing PA6 fibrils could be evaluated indirectly as indicated in [35]. First, the diameters of the PA6 globules embedded in the HDPE matrix are measured in the SEM micrographs of HDPE/PA6 blends before orientation (Figure 17.6, 1-4(a)). Then, the diameters of the fibrils in the final MFCs are measured from the respective SEM images and averaged. Supposing that the fibrils' geometry is cylindrical and that they are produced by deforming the respective PA6 spheres without the formation of voids, i.e., that the volume of the PA6 nodules at the extruder die (Figure 17.6, images 1-4(a)) and of the respective MFC fibril (images 1-4 (b)) are the same, the average length and the aspect ratio of the latter are computed. Thus, in non-compatibilized PA6reinforced MFCs the length of the reinforcing fibrils can reach $120 \mu \mathrm{m}$, whereas the maximum length in the presence of compatibilizer is ca. $40 \mu \mathrm{m}$. This would give aspect ratios of 80-200 and 40-80, respectively.

Discussing the MFCs in Figure 17.6 it is worth noting that the fibril thickness in images 1-4 (b) varies as a function of the sample composition. The question arises if the fibrils observed are of pure PA6 or also include at their interface physically or chemically bonded oriented HDPE. This question can be elucidated by X-rayscattering experiments.

2D SAXS Studies of HDPE/PA6/YP MFC It can be supposed that after the matrix isotropization stage the final composite will contain fibrillar reinforcement components embedded in a fully isotropic matrix. The presence of fibril-shaped phase is undoubtedly proved by the SEM micrographs of the UDP MFCs in Figure 17.6. Figure 17.7 represents the SAXS patterns of two HDPE/PA6/YP UDP MFC compositions: without compatibilizer (1) - 80/20/0 and with compatibilizer (2) - 70/20/10 at different temperatures. The first examination of the 2D SAXS patterns (images 1(a) and 2(a) show that both composites contain isotropic scattering of randomly distributed lamellar structures and equatorial scattering maxima attributable to lamellar crystals oriented parallel to the horizontal fiber direction. The isotropic ring and the oriented maxima display similar long spacings of $>220 \AA$. This is a clear indication that the observed oriented reflections cannot originate from the reinforcing PA6 whose $L_{B}$ values are 
(a)

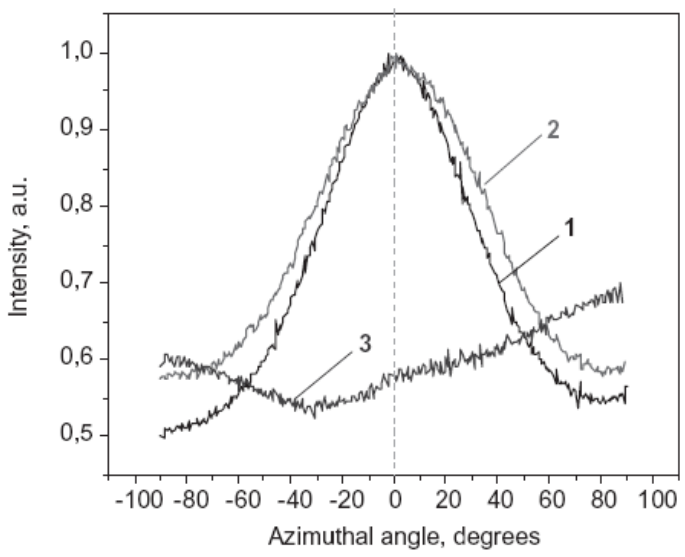

(b)

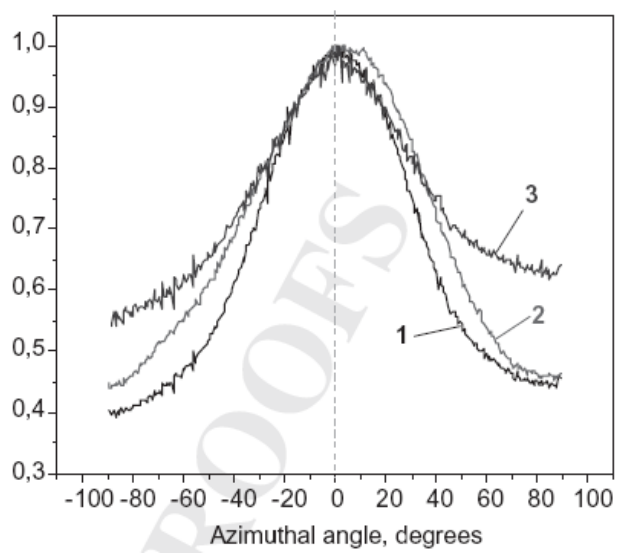

Figure 17.8 Azimuthal distribution of the scattered intensity in the 2D SAXS images of two HDPE/PA6/YP UDP MFCs: (a) 80/20/0; (b) 70/20/10. 1 - Initial MFC at 30 ${ }^{\circ}$ C; 2 - in beam heating at $160^{\circ} \mathrm{C} ; 3-$ at $30^{\circ} \mathrm{C}$ after heating to $160^{\circ} \mathrm{C}$. The dashed line indicates the fiber direction [47].

typically between 70 and $90 \AA$ [46]. Consequently, it can be supposed that a fraction of the HDPE matrix material has crystallized upon the oriented PA6 fibrils thus forming a transcrystalline layer (TCL) at the interface.

Without a special treatment it is impossible to observe at the same time the HDPE and PA6 scattering in patterns 1(a) and 2(a) because of the strong differences in the scattering intensities. Heating the two UDP MFC samples at $160{ }^{\circ} \mathrm{C}$ eliminates the HDPE scattering and reveals the oriented PA6 reflections (images 1(b) and 2(b)). Cooling back to $30^{\circ} \mathrm{C}$ causes the HDPE matrix to recrystallize. This process takes place differently in the two MFCs under investigation.

The oriented HDPE TCL in 70/20/10 MFC at $30{ }^{\circ} \mathrm{C}$ after the selective melting of the matrix maintains its equatorial orientation (Figure 17.7, 2(c)), whereas in the 80/20/0 system it rotates by $90^{\circ}$ and appears at the meridian (Figure 17.7, 1(c)). Isotropic scattering was also present in the two patterns.

This reorientation of the HDPE scattering of is better observed if azimuthal cuts of the above patterns are performed (Figure 17.8). The curve of the noncompatibilized sample (Figure 17.8(a)) clearly shows that after recrystallization the peak of intensity is not at 0 ० (i.e. along the fiber axis) but at -90 or $90^{\circ}$. In the compatibilized sample (b) the azimuthal distribution of scattered intensity remains the same at $30{ }^{\circ} \mathrm{C}$ and at 30 after $160^{\circ} \mathrm{C}$. It is noteworthy that this reorientation of the lamella that takes place in the noncompatibilized samples is not accompanied by chain direction reorientation, i.e. the chain direction of PA6 and that of the oriented HDPE fraction continue to coincide, as in the starting image at $30^{\circ} \mathrm{C}$. This effect will be discussed in the next section dedicated to the WAXS studies.

To make a distinction between the two fractions of HDPE, the subtraction procedure described by Nogales et al. [51] was used. The 2D WAXS patterns were first corrected for the incident beam intensity and then the empty chamber scattering was subtracted. It was assumed that the total scattered intensity could be separated into two contributions: (i) the isotropic contribution from the amorphous chains and the non-oriented crystals, being directly proportional to the azimuthally independent component of the total scattered intensity, and (ii) the oriented contribution from all oriented (with varying degree of orientation) scatterers calcu - 
(a)

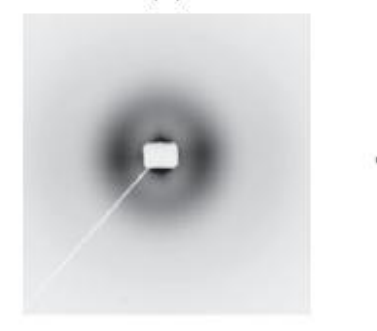

(b)

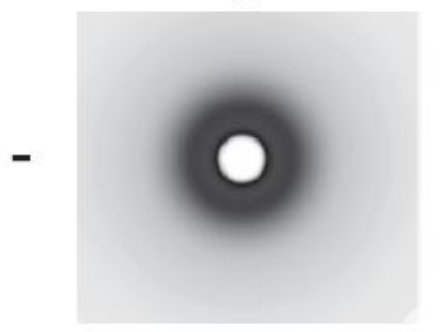

(c)

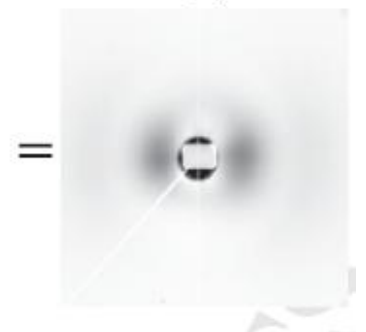

Figure 17.9 Deconvolution procedure of the SAXS pattern of the 75/20/5 UDP MFC. (a) - original SAXS image;(b) intensity pattern of the isotropic scattering; (c) intensity pattern of the oriented scatters obtained by subtraction $(a)-(b)$ [12]. The fiber axis is horizontal [47].

lated by subtracting the azimuthally independent component from the total scattered intensity. To determine the azimuthally independent intensity and to perform the said subtraction, a subroutine incorporated into the POLAR 2.7.1 X-ray software was used [52]. Thus, Figure 17.9(a) shows the pattern of the total scattering of the 75/20/5 MFC composition at $30{ }^{\circ} \mathrm{C}$. The computer-generated 2D image of the isotropic intensity is presented in Figure 17.8(b), and the resulting image obtained after (a) - (b) subtraction, corresponding to the oriented scatterers is shown in Figure 17.10(c). As seen from the latter, the said procedure not only separates the two HDPE components, but also reveals clearly the oriented PA6 fraction located along the equator.

In Figure 17.10, a 3D visualization of the initial pattern (a) and that of the oriented scattering (b) for the same 75/20/5 composition is given. Image (b) shows better the PA6 contribution to the oriented part of the scattering, pointed by the arrows.

Table 17.3 contains the HDPE and PA6 $L_{B}$ values determined from the scattering patterns of six UDP MFCs with different HDPE/PA6/YP compositions. It can be seen that in the absence of compatibilizer, there are no significant differences between the long spacings values of HDPE lamellae located in the bulk (isotropic) and those of the oriented HDPE lamellae in the transcrystalline layer (oriented). Introducing YP compatibilizer results in smaller long periods in the oriented HDPE fraction, while that of the bulk matrix fraction remains as in the noncompatibilized compositions. Only in the 65/30/5 UDP MFC the distance between the oriented
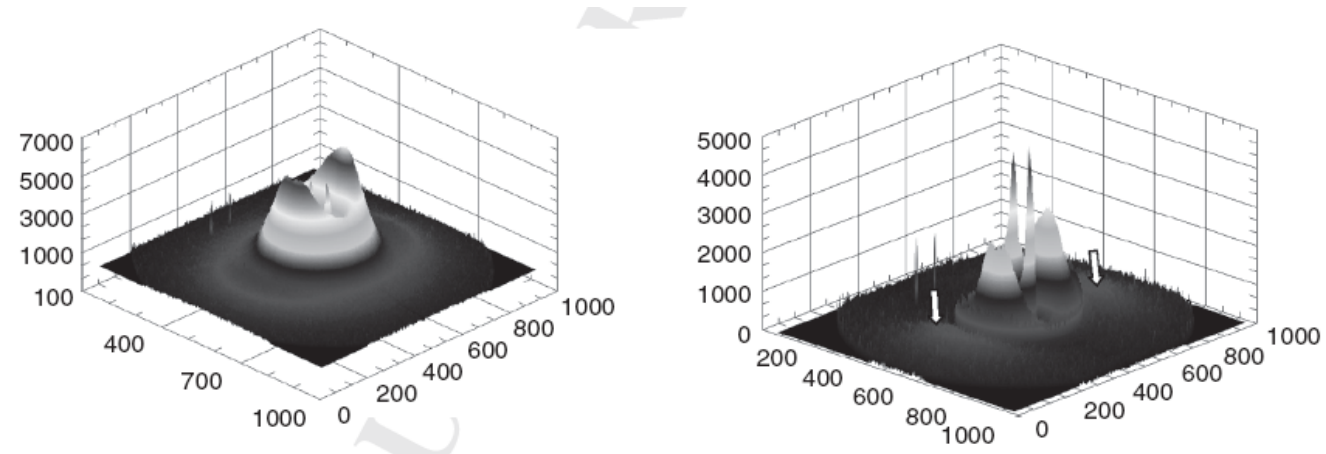
Table 17.3 Long spacing values of the HDPE/PA6/YP UDP composites at $30^{\circ} \mathrm{C}$ without $\left(\mathrm{L}_{\mathrm{B}}\right)$ and with $\left(\mathrm{L}_{\mathrm{B}}^{*}\right)$ deconvolution.

\begin{tabular}{|c|c|c|c|c|c|c|}
\hline \multirow[b]{2}{*}{$\mathrm{N}^{\circ}$} & \multirow{2}{*}{$\begin{array}{l}\text { HDPE/PA6/YP } \\
\text { composition }\end{array}$} & \multicolumn{2}{|c|}{$L_{B}, \AA$} & \multicolumn{3}{|c|}{$L_{B} *, \AA$} \\
\hline & & PA6 & HDPE total & HDPE-iso & HDPE-orient. & PA6 orient. \\
\hline 1 & $90 / 10 / 0$ & 100.5 & 223 & 224 & 222 & 95 \\
\hline 2 & $80 / 20 / 0$ & 90 & 229 & $225(231)$ & 222 (225) & 86 \\
\hline 3 & $77.5 / 20 / 2.5$ & 94 & 221 & 224 & 211 & 91 \\
\hline 4 & $75 / 20 / 5$ & 94 & 220 & 224 & 213 & 92 \\
\hline 5 & $70 / 20 / 10$ & 87 & 215 & $225(245)$ & $210(214)$ & 88 \\
\hline 6 & $65 / 30 / 5$ & 82 & 236 & 223 & 231 & 77 \\
\hline
\end{tabular}

Note: The values in parentheses were obtained after recrystallization of the HDPE by in beam heating to $160^{\circ} \mathrm{C}$ followed by cooling down to $30^{\circ} \mathrm{C}$. [47].

HDPE lamellae is bigger than that of the isotropic fraction. Most probably, this could be explained as a result of a higher amount of PA6 in this composition. As regards the PA6 $L B$ values, they vary in the $77-95 \AA$ interval. The PA6 long period of $77 \AA$ in the $65 / 30 / 5$ composition is the closest to the value of the neat oriented PA6 [46].

As mentioned above, after recrystallization, the HDPE fraction in the non-compatibilized and compatibilized samples orients in different ways - in the first case the scattering maxima appeared on the meridian, while in the second maintained their position on the equator. As seen from Table 17.3, in both 80/20/0 and 70/20/10 samples, an increase of the $L_{B}$ values of the isotropic HDPE - in the presence and in the absence of compatibilizer - was observed after matrix recrystallization (the data in parentheses).

A significant improvement of the amount and quality of structural information extracted from the oriented part of the scattering in the UDP SAXS patterns (Figure 17.8(c)) can be achieved by image reconstruction followed by computation of the respective Chord Distribution Function, CDF, as suggested in a series of recent papers by Stribeck et al. [53, 54]. An example of this treatment is given in Figure 17.11 for the two UDP MFC samples without and with compatibilization. The image reconstruction comprises background correction and calibration for beam intensity, followed by filling of the 'blind' areas behind the beam stop and its wire. Thereafter, the computation of the $\mathrm{CDF}$ is performed. It is worth noting that all these procedures are carried out automatically using the pv Wave ${ }^{\circledR}$ programming environment [55].

Analyzing the reconstructed patterns of the oriented scattering in the two samples under investigation, it can be concluded that the respective $L B$ values of HDPE (oriented and isotropic) as well as of the PA6 almost coincide with those in Table 17.3. Interesting information can be extracted from the quite complex CDF functions. It is important to note that the CDF images reflect mostly the structure of the HDPE-oriented TCL and to a lesser extent that of the PA6 fibrils. Moreover, the CDF reveals more features than the respective SAXS pattern, being its transformation to the real space. Thus, in the as-prepared UDP MFC without compatibilization (80/20/0 sample), the HDPE TCL forms a microfibrillar system with first and second order long periods, with the HDPE domains being side-by-side and not shifted. Introducing compatibilizer (70/20/10 sample) maintains these structural features but results in the appearance of tilted oriented HDPE domains. The differences between the oriented SAXS fractions in compatibilized and non-compatibilized UDP MFC materials and especially in the corresponding oriented precursors become better pronounced during a simultaneous SAXS/straining experiment. As it was demonstrated recently [55], the automated procedures described above allow the processing of hundreds of data frames associating the structural features with the simultaneously obtained mechanical behavior in stretching or load cycling modes. 
Figure 17.11 Reconstructed SAXS patterns (oriented scattering) of two UDP MFC materials and their respective chord distribution functions. Fibril axis is vertical. The CDF function is presented in absolute values (both positive and negative faces in one image). For more details see the text.

2D WAXS Studies of HDPE/PA6/YP MFC The SAXS studies of UDP MFC materials gave evidence that the reinforcing fibrils most probably have a layered, coaxial structure: a core of oriented PA6 and a shell of oriented, transcrystalline HDPE. The WAXS measurements supported this hypothesis.

The visual inspection of the 2DWAXS patterns of UDP MFCs (Figure 17.12) shows that the crystallographic characteristics of HDPE and PA6 are very similar leading to a strong overlapping of the respective diffraction peaks. Nevertheless, one can notice that at $30^{\circ} \mathrm{C}$ there is a co-existence of isotropic Debye rings and crystalline reflections oriented parallel to the horizontal fibril direction. At $160^{\circ} \mathrm{C}$ the $\mathrm{HDPE}$ reflections change into a diffuse amorphous halo revealing the oriented PA6 reflections.

To separate the contribution of the isotropic and oriented crystalline fractions and to study their origin, the same subtraction procedure as with the SAXS patterns was applied. Figure 17.13 exemplifies this treatment for the 80/20/0 (a) and 70/20/10 (b) HDPE/PA6/YP UDP MFCs showing the starting real 2DWAXS patterns (left), the computer-generated isotropic part of the scattered intensity (center) and the resulting 2D WAXS images of the oriented part after subtraction (right).

Subtracting the isotropic crystalline and amorphous fractions allows the outlining of the oriented crystalline reflections that are otherwise undetectable. Together with the expected oriented PA6 reflections in the right images in Figure 17.13, one observes also clear reflections of the oriented matrix. The two weak equatorial arcs belong to the (200) and (002/202) planes of PA6 and the other two, more intense equatorial reflections belong to the (110) and (200) planes of orthorhombic unit cell of HDPE. This is one more indication for epitaxial crystallization of matrix material upon the reinforcing fiber, whereby the chain direction in the matrix crystals coincides with that in the reinforcing PA6 fibrils. Judging from Figure 17.13, this is valid for both selected samples - non-compatibilized (a) and compatibilized (b). 


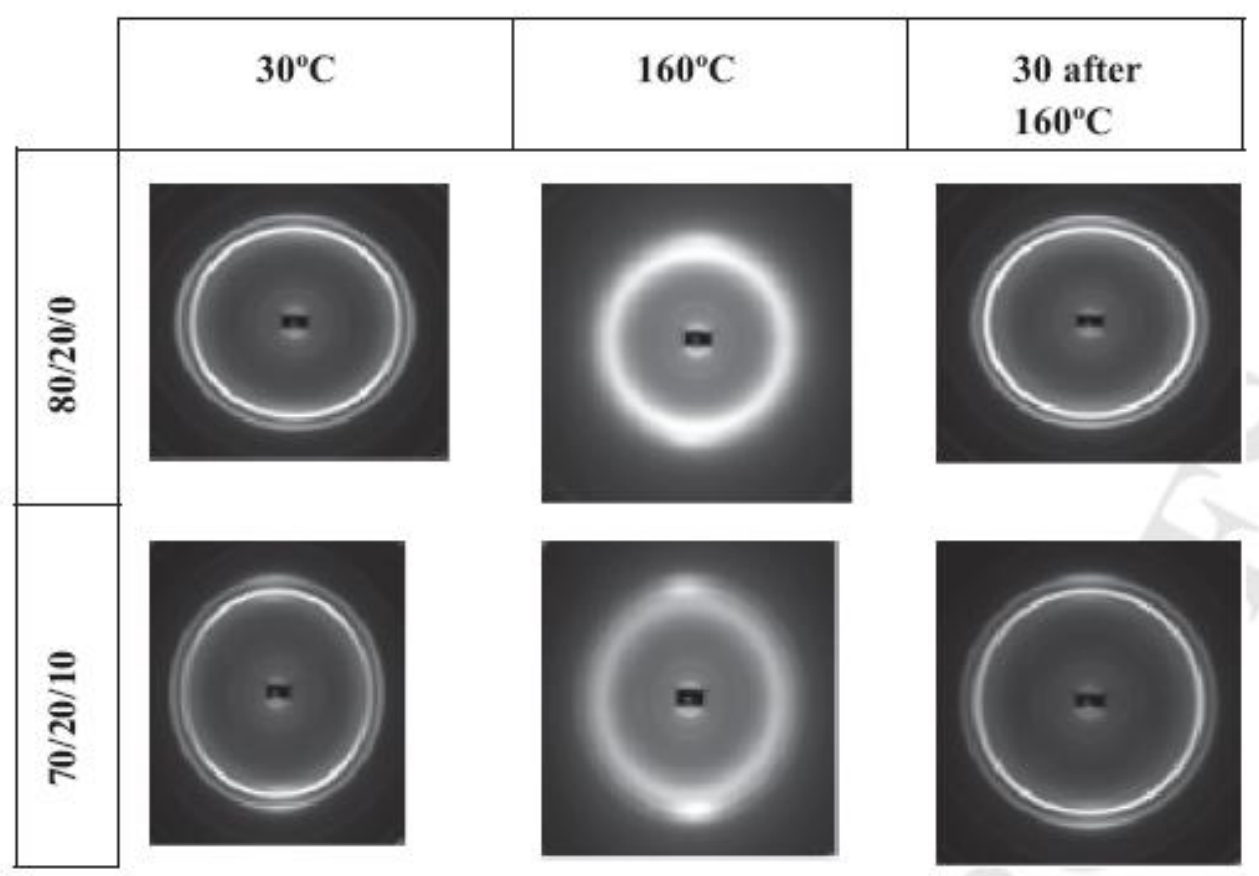

Figure 17.12 2D WAXS patterns of HDPE/PA6/YP microfibrilar composites taken at various temperatures. Fibril direction is horizontal [47].

(a)

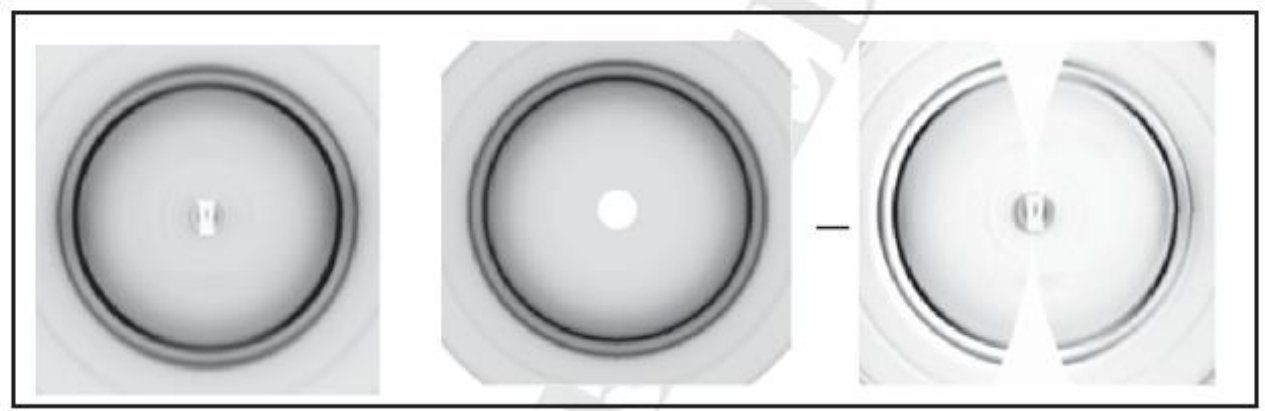

(b)

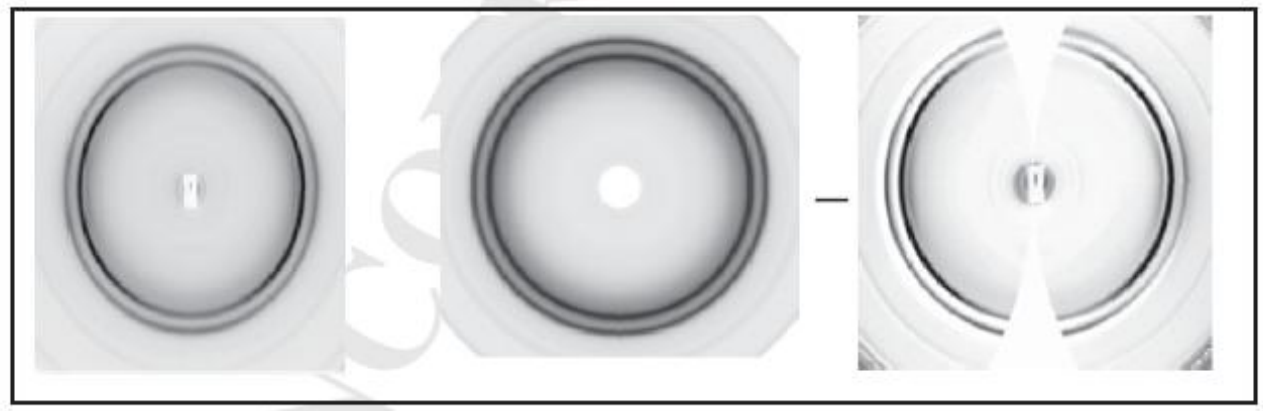

Figure 17.13 Example of the analysis of theWAXS patterns at $30{ }^{\circ} \mathrm{C}$ of UDP MFCs: Left - total scattered intensity; Center: calculated isotropic intensity; Right: oriented scattered intensity. (a) 80/20/0 and (b) - 70/20/10. The fiber axis is vertical [47.] 

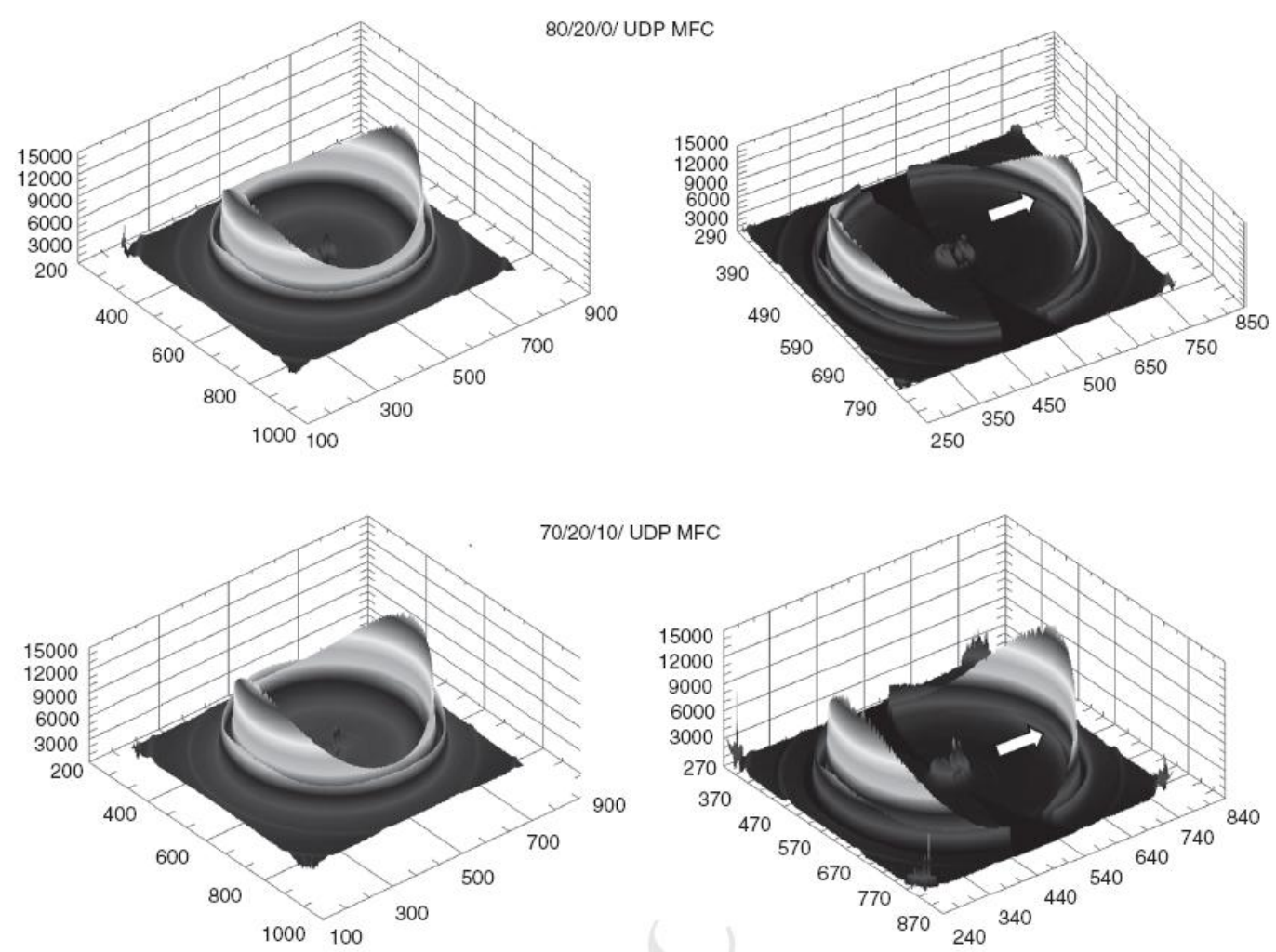

Figure 17.14 3D WAXS patterns of UDP MFCs before (left) and after (right) the subtraction of the azimuthally independent component of the total scattered intensity. The white arrows point at the (200) reflections of $\alpha$ PA6 [47].

Figure 17.14 shows the 3D images of the real WAXS patterns before treatment (left) and of the oriented scattering after subtracting (right) of the same two MFCs. The white arrows indicate the position of the $\alpha$-PA6 (200) reflection. This representation shows better the anisotropy of the HDPE (110) and (200) diffractions.

For a quantitative evaluation of oriented and isotropic parts of the total scattered intensities, the respective 2DWAXS patterns were integrated in the $0-180^{\circ}$ range to get the 1DWAXS profiles, which were afterwards fitted by Gaussian peaks. The results from peakfitting applied in the 80/20/0 MFC sample are presented in Figure 7.15(a) and (b). The deconvolution of the integral profile of the oriented part clearly shows the (110), (200) and (210) contributions of the HDPE (Figure 17.15(a), the shaded reflections)) and also the four crystalline reflections of $\alpha$ - and $\gamma$ PA6. The peak-fitting of the isotropic part displayed crystalline reflections (110), (200) and (210) of the HDPE matrix only and the amorphous halos of PA6 and HDPE, respectively (Figure 17.15(b)). Based on the angular position of the reflections, the d-spacings $(d h k l)$ of the corresponding planes were calculated. A quantitative evaluation of the peak-fitting results for two representative MFCs - without (80/20/0) and with compatibilization (70/20/10), as well as data for d-spacings are given in Table 17.4. 

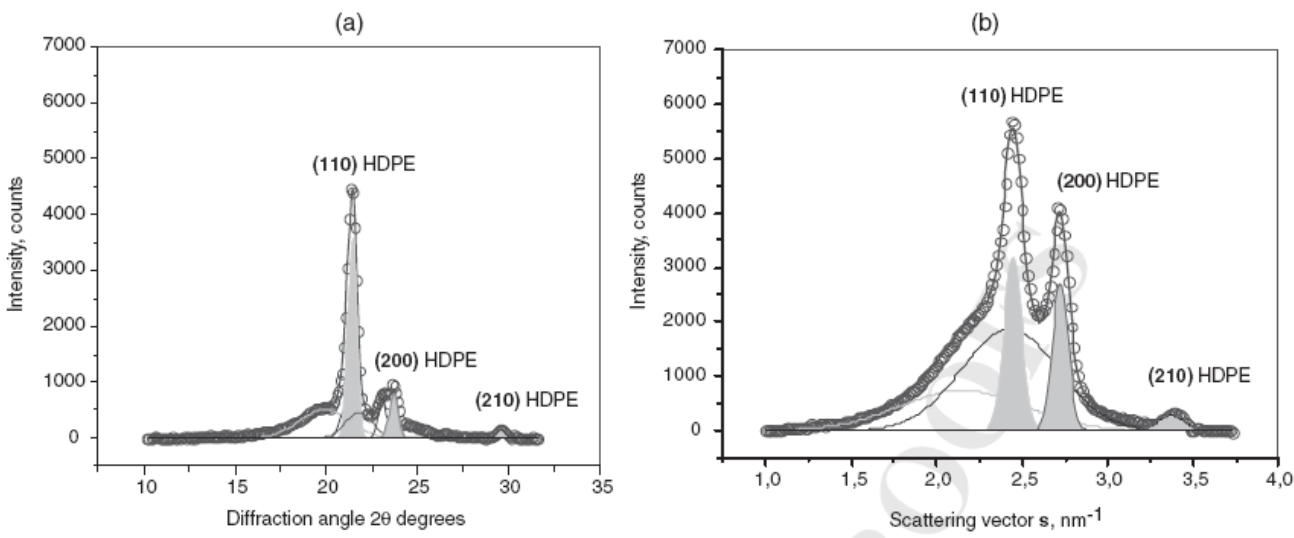

Figure 17.15 1D WAXS profiles of the 80/20/0 HDPE/PA6/YP UDP MEF exemplifying the peak-fitting of the oriented scattering (a) and of the isotropic EWAXS scattering (b). The pattern in (a) was obtained after subtraction of $(b)$ from the initial WAXS pattern with the total scattered intensity [47]

Table 17.4 Results from the deconvolution of the oriented and isotropic part of 2D WAXS patterns of selected HDPE/PA6/YP UDP MFC.

\begin{tabular}{|c|c|c|c|c|c|c|}
\hline \multirow[b]{3}{*}{ WAXS Reflections } & \multicolumn{6}{|c|}{ HDPE/PA6/YP } \\
\hline & \multicolumn{3}{|c|}{$80 / 20 / 0$} & \multicolumn{3}{|c|}{$70 / 20 / 10$} \\
\hline & $2 \theta, \mathrm{deg}$. & Content, \% & $d_{h k l}, \AA$ & $2 \theta$, deg. & Content, \% & $d_{h k l}, \AA$ \\
\hline \multicolumn{7}{|c|}{ Oriented part of WAXS intensity } \\
\hline$(200)-\alpha$ PA 6 & 19.90 & 28.5 & 4.34 & 19.92 & 28.7 & 4.34 \\
\hline$(001)-\gamma$ PA6 & 21.05 & 6.6 & 4.11 & 21.35 & 7.6 & 4.07 \\
\hline (110) - HDPE & 21.44 & 34.9 & 4.03 & 21.33 & 38.2 & 4.05 \\
\hline$(200)-\gamma$ PA6 & 21.79 & 13.7 & 3.97 & 21.66 & 7.6 & 3.99 \\
\hline$(002) /(202)-\alpha$ PA6 & 23.09 & 6.9 & 3.75 & 22.99 & 6.9 & 3.76 \\
\hline (200) - HDPE & 23.69 & 7.9 & 3.65 & 23.74 & 9.1 & 3.65 \\
\hline (210) - HDPE & 29.61 & 1.5 & 2.94 & 29.50 & 1.9 & 2.95 \\
\hline PA6 fraction, $\%$ & \multirow{2}{*}{\multicolumn{3}{|c|}{$\begin{array}{l}55.7 \\
44.3\end{array}$}} & \multirow{2}{*}{\multicolumn{3}{|c|}{$\begin{array}{l}50.8 \\
49.2\end{array}$}} \\
\hline HDPE fraction, $\%$ & & & & & & \\
\hline \multicolumn{7}{|c|}{ Isotropic part of WAXS intensity } \\
\hline (110) - HDPE & 21.13 & 14.6 & 4.09 & 20.97 & 9.8 & 4.12 \\
\hline (200) - HDPE & 23.56 & 11.4 & 3.67 & 23.48 & 12.6 & 3.69 \\
\hline (210) - HDPE & 29.29 & 1.9 & 2.96 & 29.24 & 1.3 & 2.97 \\
\hline
\end{tabular}

Notes: In the isotropic part of the WAXS intensity the crystalline reflections only are included. The difference to $100 \%$ will give the content of the amorphous HDPE and amorphous PA6. $d_{h k}$ is the dspacing of the respective crystalline plane. The oriented reflections are considered $100 \%$ crystalline [47].

11 
UDP MFC 70/20/10
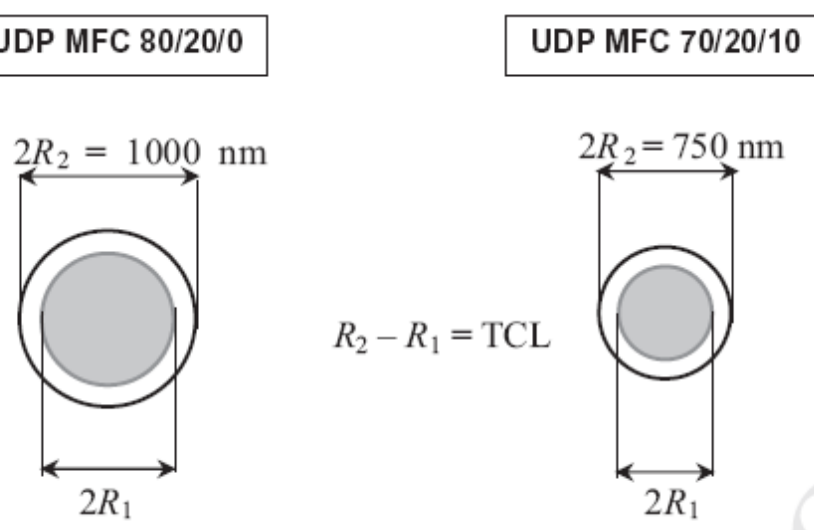

Figure 17.16 Schematic presentation of the fiber cross-sections of 80/20/0 and 70/20/10 UDP MFCs.

From Figure 17.15 and Table 17.4 it can be seen that a significant part of the HDPE matrix is able to crystallize oriented along the PA6 fiber, thus forming a transcrystalline layer in such a way that the chain directions of the two polymers coincide. The rest of the matrix, situated in the bulk, crystallizes isotropically. The relation between the content of the PA6 fibrils and the oriented part of the HDPE matrix (the crystalline fraction) is almost 1.03:1.00 in the 70/20/10 MFC and 1.26:1.00 in the 80/20/0 system. This means that in the presence of compatibilizer a larger part of the HDPE is included in the transcrystalline layer without changing considerably its crystallographic characteristics. Based on the d-spacing values it can be concluded that the HDPE unit cell is slightly larger in the bulk, as compared to that in the oriented transcrystalline layer.

The data about the PA6 and HDPE fraction in the oriented scattering can be used to obtain an estimate of the TCL thickness in UDP MFC materials. From the SEM studies (Figure 17.6) one can estimate the average visible thickness of the reinforcing fibrils in the MFC composition. Let us take samples 1(b) and 4(b) and suppose that the fibrils are cylindrical with a PA6 core (with a diameter 2R1) being uniformly coated by coaxial transcrystalline layer of HDPE whose thickness is given by $\mathrm{R} 2-\mathrm{R} 1$. Therefore, the visible diameter of the fibril estimated form SEM will be R2. Figure 17.16 gives a schematic view of the cross-sections in the two selected UDP MFCs - without and with compatibilization.

Whenever X-rays are interacting with matter, their main partners are the electrons in the studied sample. Thus X-ray scattering is probing the distribution of electron density, $\rho(\boldsymbol{r})$, inside the material. In the field of WAXS, $\rho(\boldsymbol{r})$ is identical to the average electron density, $\rho$. For a given material or specific phase within a material, $\rho$ is calculated as [8]:

$$
\rho=N_{A} \frac{Z_{M}}{M_{M}} \rho_{m}
$$

Here, $\rho m$, is the respective average mass density, NA is the Avogadro's number $(6.022 \times$ $\left.10^{23} \mathrm{~mol}^{-1}\right), Z_{M}$ is the number of electrons per molecule or monomer unit and $M_{M}-$ the molecular weight of molecule or monomer unit. Logically, the intensity of the radiation diffracted by either the PA6 or HDPE component will be proportional to the volume of this phase $V_{i}$ and the respective average electron density $\rho_{i}$ :

$$
I_{S}^{i} \sim V_{i} \cdot \rho_{i}
$$


If we denote by $V_{P A 6}$ the volume of the PA6 core, it can be written that

$$
\begin{aligned}
V_{P A 6} & =\pi L R_{1}^{2} \quad \text { and } \\
V_{T C L} & =\pi L\left(R_{2}^{2}-R_{1}^{2}\right)
\end{aligned}
$$

Combining Eq. (17.14) with (17.15) and (17.16), the following simple dependence can be deduced between the visible by SEM fibril radius $R_{2}$ and that of the PA6 core $R_{1}$ :

$$
R_{1}^{2}=R_{2}^{2} \cdot \sqrt{\frac{f}{k+f}},
$$

wherein $k=\frac{\rho_{P A 6}}{\rho_{H D P E}}$ and $f=\frac{I_{S}^{P A 6}}{I_{S}^{H D P E}}$.

Table 17.5 summarizes the structural information related to the reinforcing fibrils as revealed by SEM and WAXS methods (i.e. $2 R_{1}, 2 R_{2}$ and $R_{2}-R_{1}$ ) for MFC materials without and with compatibilization reinforced by PA6 or PA12. The same table contains also some mechanical data obtained with these composites, as well as with the neat HDPE matrix material and with the neat oriented polyamides.

It can be concluded that the formation of transcrystalline layers TCL is a common feature for all MFCs containing either PA6 or PA12. There can be a significant difference between the TCL thicknesses in PA6 and PA12 reinforced composites, as well as in the compatibilized and noncompatibilized MFCs with the same reinforcement. Compatibilization results in thinner fibrils in which not only the polyamide core, but also the TCL are finer. In PA6 reinforced MFCs the TCL is more than two times thicker than in similar HDPE/PA12/YP composites. Obviously, the TCL thickness is directly related to the mechanical performance of the MFCs, whereby the larger the thickness, the lower the properties. Thus, no matter that the $E 1$ value of neat oriented PA6 is much higher than that of PA12, in both compatibilized and noncompatibilized MFCs this values is either similar or lower in the HDPE/PA6/YP materials. At the same time, the $\sigma y$ of the HDPE/PA12/YP materials are significantly higher, irrespective of the almost coinciding values of the neat oriented polyamides. It is to be noted the superior flexural stiffness of the PA12 composites, whichmay have to do with the increased flexibility of PA12 and its better compatibility with HDPE. One has to bear in mind also that in the 80/20/0 samples the formation of TCL can be attributed to physical interactions at the matrix-fibril interface. In the 70/20/10 systems, however, it should be a result of chemical reactions between the maleic anhydride of YP and the amide groups of the polyamide [57]. It can be expected that in the latter case the TCL will include polyolefin component from the YP compatibilizer, which is different from the bulk matrix HDPE. This could be one of the possible explanations of the inferior mechanical properties of the compatibilized samples.

\begin{tabular}{|c|c|c|c|c|c|c|c|}
\hline & \multicolumn{2}{|c|}{ HDPE/PA6/YP } & \multicolumn{2}{|c|}{ HDPE/PA12/YP } & \multicolumn{3}{|c|}{ Neat materials } \\
\hline & $80 / 20 / 0$ & $70 / 20 / 10$ & $80 / 20 / 0$ & $70 / 20 / 10$ & PA6 orient. & PA12 orient. & HDPE isotropic \\
\hline $2 \mathrm{R}_{2}, \mathrm{~nm}$ & 1000 & 750 & 700 & 560 & - & - & - \\
\hline $2 \mathrm{R}_{1}, \mathrm{~nm}$ & 733 & 524 & 567 & 457 & - & - & - \\
\hline $\mathrm{R}_{2}-\mathrm{R}_{1}, \mathrm{~nm}$ & 134 & 113 & 66 & 52 & - & - & - \\
\hline$E_{1}, \mathrm{MPa}$ & 1095 & 920 & 1054 & 972 & 3180 & 2240 & 827 \\
\hline$\sigma_{y}, \mathrm{MPa}$ & 57 & 37 & 64 & 55 & 230 & 233 & 26 \\
\hline$C_{R}, \mathrm{MPa}$ & 2624 & 2294 & 3414 & 3404 & - & - & 1478 \\
\hline
\end{tabular}

Table 17.5 Dependence between the structural parameters related to the oriented reinforcing component and the mechanical behavior in various MFC materials.

Notes: $E_{1}$ is the secant modulus determined at $1 \%$ strain; $\sigma_{y}$, is the maximum stress at break and $C_{R}$ is the three point support flexural stiffness determined according to Nunes et al [56]. 


\subsubsection{Immiscible Polymer Blends}

\subsubsection{Materials and Sample Preparation}

A blend of polypropylene/polystyrene (PP/PS) was selected for the investigations, comprising a semicrystalline and amorphpous polymers. The two polymers PP/PS were mechanically blended in a tumbler mixer, in the ratio of 70/30 wt $\%$. The blend was then directed injection molded in the form of small tensile specimens. High back pressure was used to promote a better mixing between both components.

\subsubsection{Experimental Techniques}

The structure of the injection molded PP/PS specimens was characterized by SEM and WAXS techniques. The specimens were also stretched in a tensile machine until different strain levels (of 5, 10, 15, 20 and 25\%) in the plastic regime (inducing permanent deformation, but allowing molecular relaxation). The structure of the deformed specimens were then analyzed by 2D WAXS and SAXS experiments (not simultaneously), which allowed the characterization of the structure evolution of the semicrystalline polymer (PP) and damage occurring by nanocavitation phenomena (e.g. at the polymer interfaces) upon stretching. The sample-to-WAXS detector distance was of $13.4 \mathrm{~cm}$ and the sample-to-SAXS distance of $280 \mathrm{~cm}$.

\subsubsection{Results and Discussion}

Structure Development During Processing Figure 17.17 shows the solid-state morphology of the PP/PS injection molded blend. In Figure 17.17(a) are presented the polarized light microscopy (PLM) and scanning electron microscopy (SEM) images of the blend. The injection molded specimen evidences a typical skin-core microstructure. In the skin layer, the disperse component (PS) features very thin and highly elongated strands (in the flow direction, FD) due to the high deformation rates applied during molding. In the core, this disperse component is constituted by particles of different shapes (e.g. spherical, elongated fibers) of higher dimensions, mostly oriented transversely to FD [58].

Figure 17.17(b) shows the WAXS and SAXS patterns of the blend. These are average patterns through half thickness of the molded specimens. From the WAXS pattern, the molded PP/PS blend features an oriented PP crystalline phase, expectantly in the skin region. A bimodal orientation distribution of the crystalline phase of $\mathrm{PP}$ is revealed, with a relatively high $\mathrm{a}^{*}$-axis oriented component [59]. This may arise from the development of transcrystalline structures growing on the highly oriented PS component. The SAXS pattern reveals a typical shish-kebab structure, with lamellae perpendicular to FD and growing in lamellar stacks along FD. The central black region of the SAXS patterns suggest that the PP/PS blend may show some nanosized voids, which may be originated at the interface between the two polymers.

Structure Evolution During Stretching The structure evolution and damage during solid-state stretching of immiscible (non-compatibilized) PP/PS blend was also investigated by X-ray scattering techniques. Compared with the neat PP, the PP/PS blend shows substantially reduced deformation capabilities. Figure 17.18 presents the obtained stress-strain curve, and correspondent WAXS and SAXS patterns at given strain levels. 


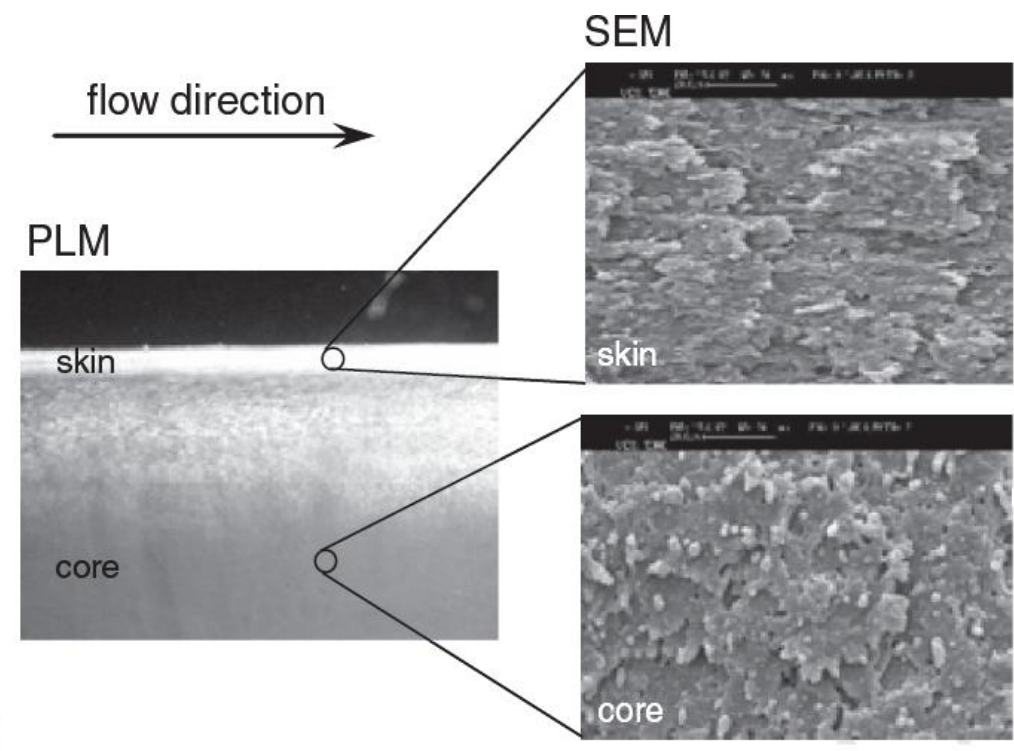

(b)
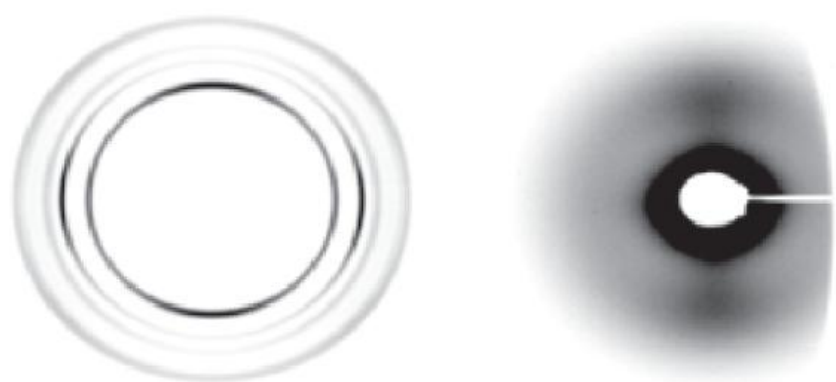

Figure 17.17 Morphology of the injection molded PP/PS blend: (a) polarized (left) light and scanning electron (right) microscopy images; (b) WAXS (left) and SAXS (right) patterns.

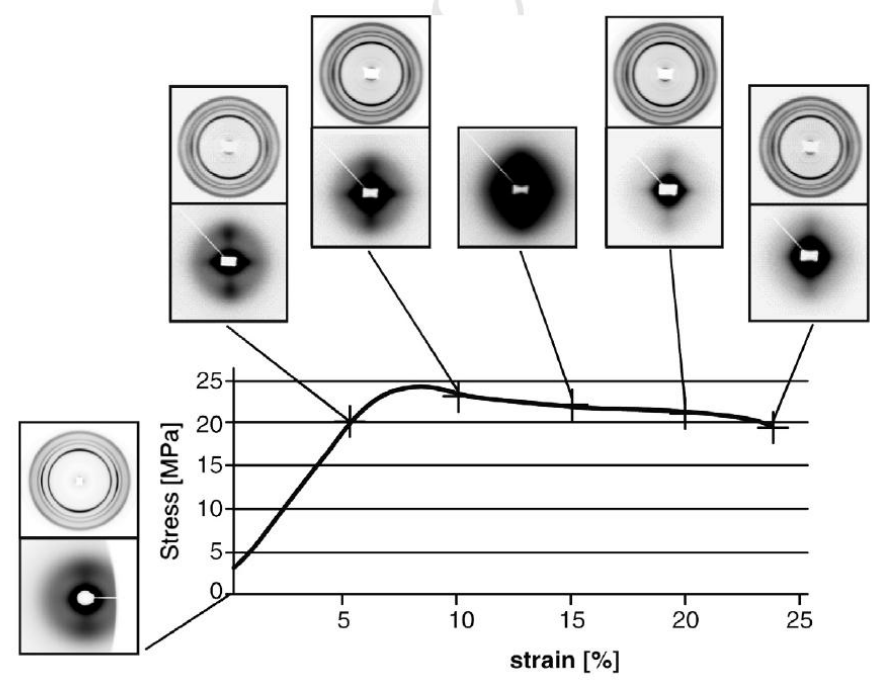

Figure 17.18 Stress-strain curve of PP/PS blend and WAXS and SAXS patterns at given strain levels. 
The PP matrix shows little evolution of its crystalline structure during stretching, with a slight increase on the level of crystalline phase orientation in the stretching direction, SD. This appears to take place at low strains during the initial linear zone of the stress-strain curve (up to $\varepsilon=0.05)$. The crystalline lamellar structure is progressively and partially destroyed on the course of deformation (reduction on the intensity of the two vertical lobules of the SAXS patterns). Significant voiding also occurs during deformation. Voids seem to be nucleated parallel to $\mathrm{SD}(\varepsilon=0.05)$, presumably at the interfaces between the two components. Then they increase laterally, growing mainly perpendicular to SD $(\varepsilon=0.10)$. At larger strain levels these voids become bigger and more elongated perpendicular to $\mathrm{SD}(\varepsilon=0.15)$. In the meanwhile, the void size increases so that SAXS could not detect them and the SAXS pattern reduces its size $(\varepsilon$ $>0.15)$, but smaller voids continue to grow $(\varepsilon=0.20)$, until complete specimen failure. Concomitantly, the stress reaches a maximum level (at $\varepsilon=0.08$ ) that progressively decreases until break. It should be remembered that both PP and PS are immiscible and no compatibilizer was used. The use of a compatibilizer should expectantly change the deformation mechanism $[27,60]$.

\subsubsection{Non-conventional Molding of PP Nanocomposites}

Shear controlled orientation in injection molding (SCORIM) is a non-conventional injection molding techniques where high levels of shearing are applied to the molten polymer during the solidification phase [61]. This develops high level of molecular orientation. When processing clay-based polymer nanocomposites, these high shearings may be beneficial for achieving a high level of clay orientation and a better exfoliation and dispersion of the nanoclays, thus imparting an improved mechanical behavior of the moldings.

\subsubsection{Materials and Sample Preparation}

PP was reinforced with different amounts of incorporation of nanoclay (montmorillonite, MMT): $0,0.5,1,3,5$ and $10 \mathrm{wt} \%$. The PP and the nanoclays were mechanically blended in a tumbler mixer, and then directed injection molded in the form of a rectangular bar. High back pressure was used during the plasticating phase of the injection molding cycle in order to promote a better mixing.

\subsubsection{Experimental Techniques}

The molding microstructures were characterized by polarized light microscopy (PLM), scanning and transmission electron microscopy (SEM and TEM, respectively) and WAXS. Charpy impact tests were performed at room temperature $\left(23^{\circ} \mathrm{C}\right)$. Fracture surfaces were observed by scanning electron microscopy (SEM).

\subsubsection{Results and Discussion}

Processing conditions and composition modify markedly the microstructure of molded PP nanocomposites. Figure 17.19 shows the effect of incorporation of MMT for fixed processing conditions (but expectantly for different thermomechanical environments due to the increase onmelt viscosity by the incorporation of MMT). The addition of MMT affects the microstructure of the mouldings (e.g. skin-core structure, the development of semicrystalline morphologies). With increased content of MMT the microstructure becomes coarser and more multi-laminated. The MMT acts as a morphology director, affecting the structure development during processing.

Figure 17.20 represents the SEM image of the external layer of the $\mathrm{PP}+5 \%$ nanoclay filled material system. In these images the flow direction is normal to the scanned surface, pointing outwards of the paper surface. A good dispersion of the MMT agglomerates in the polymer matrix was achieved. These agglomerates, with few micrometers of length, have a platelet-like shape. They are all well aligned as response to the applied shear level during processing. SCORIM technique was capable of shaping the MMT agglomerates into platelets, of well dispersing them in the polymeric matrix, and of orienting them in the flow direction. 

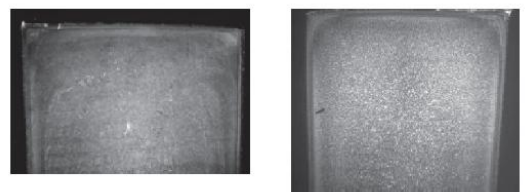

$\mathrm{PP}+0 \% \mathrm{MMT}$

$\mathrm{PP}+0.5 \% \mathrm{MMT}$

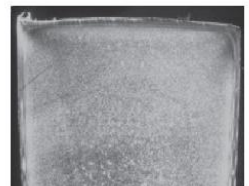

$\mathrm{PP}+1 \% \mathrm{MMT}$

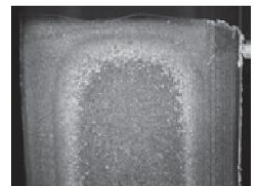

$\mathrm{PP}+3 \% \mathrm{MMT}$

Figure 17.19 Microstructure of the SCORIM moldings with different percentage of incorporation of nanoclay (MMT).The cuts are perpendicular to the bar length (or to the flow direction).

Figure 17.21 shows the variation of the impact toughness of the moldings with increased content of MMT. In the same graph are also presented the correspondent 2D-WAXS patterns. The fracture energy is maximized for an incorporation of $5 \mathrm{wt} \%$ of MMT, with gains of more than $100 \%$ in the total absorbed energy. The addition of MMT does not change significantly the flexural modulus (not shown), but enhance greatly the impact toughness. Furthermore, low melt temperatures and high shearing times lead to the best mechanical performance. The mechanical properties of PP are determined by the incorporation of nanoclays and by the processing conditions, both being highly interrelated.

The WAXS patterns reveal that the skin layer of neat PP moulding shows a small level of crystalline phase orientation. This orientation increases with the incorporation of MMT. Figure 17.22 presents the equatorial I-2 $2 \theta$ scan for different percentage of incorporation of MMT. Adding MMT induces the formation of $\beta$-phase PP (reflection at $2 \theta=16.2^{\circ}$, not present on the neat PP. This phase shows an improved toughness than the more common $\alpha$-phase [62]. Simultaneously, the crystallinity index (not shown) decreases with the incorporation of MMT. The incorporation of MMT has several effects: (i) increases the level orientation of the crystalline phase; (ii) induces the formation of $\beta$-phase PP; and (iii) reduces the degree of crystallinity. The nanoclay acts as morphology directors, thus determining the mechanical response of the moldings.

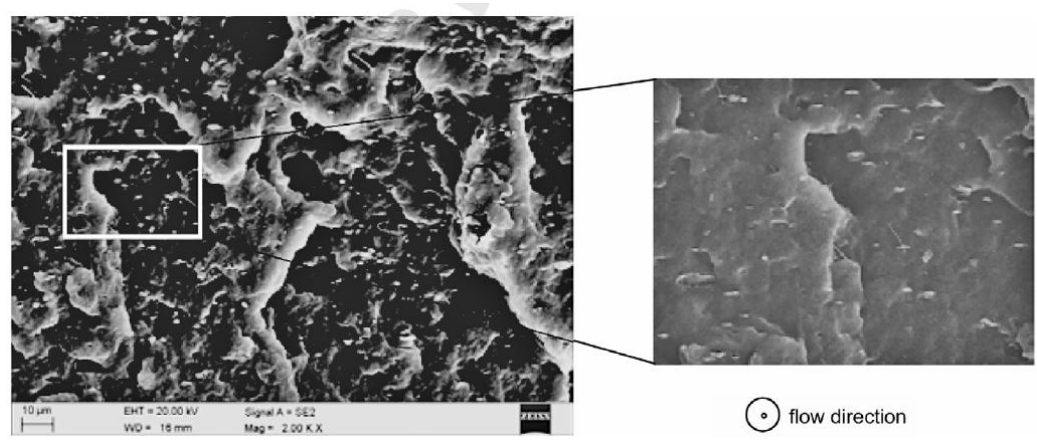

Figure 17.20 Scanning electron microscopy image of PP filled with 5\% of MMT. The flow direction points outwards the paper surface. 


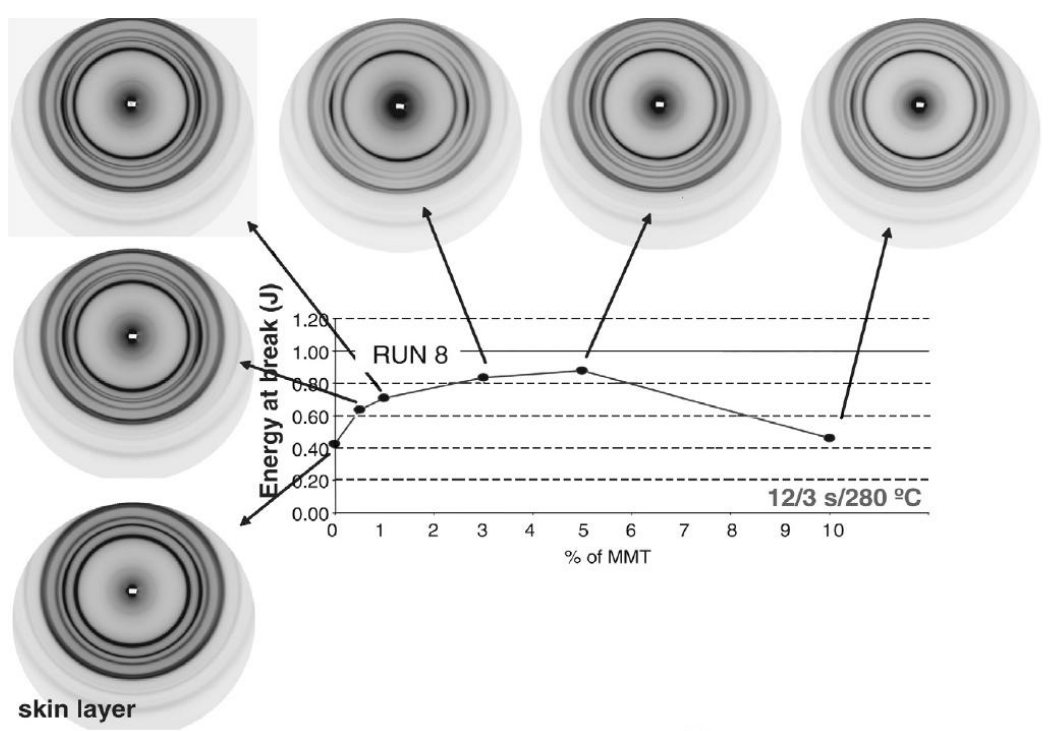

Figure 17.21 Toughness of PP as function of \% of incorporation of MMT nanoclay (SCORIM processing conditions: 12 strokes, stroke time of 3 s and melt temperature of $280^{\circ} \mathrm{C}$ ). The shown WAXS patterns are from the skin layer of the injection molded impact bars.

\subsubsection{Stretching of Nanoclay PET Nanocomposite}

Polymer nanocomposites have received a lot of attention recently because of their improved performance, namely their enhanced mechanical behavior [63]. However, the mechanisms underlying these enhancements are still not well understood.

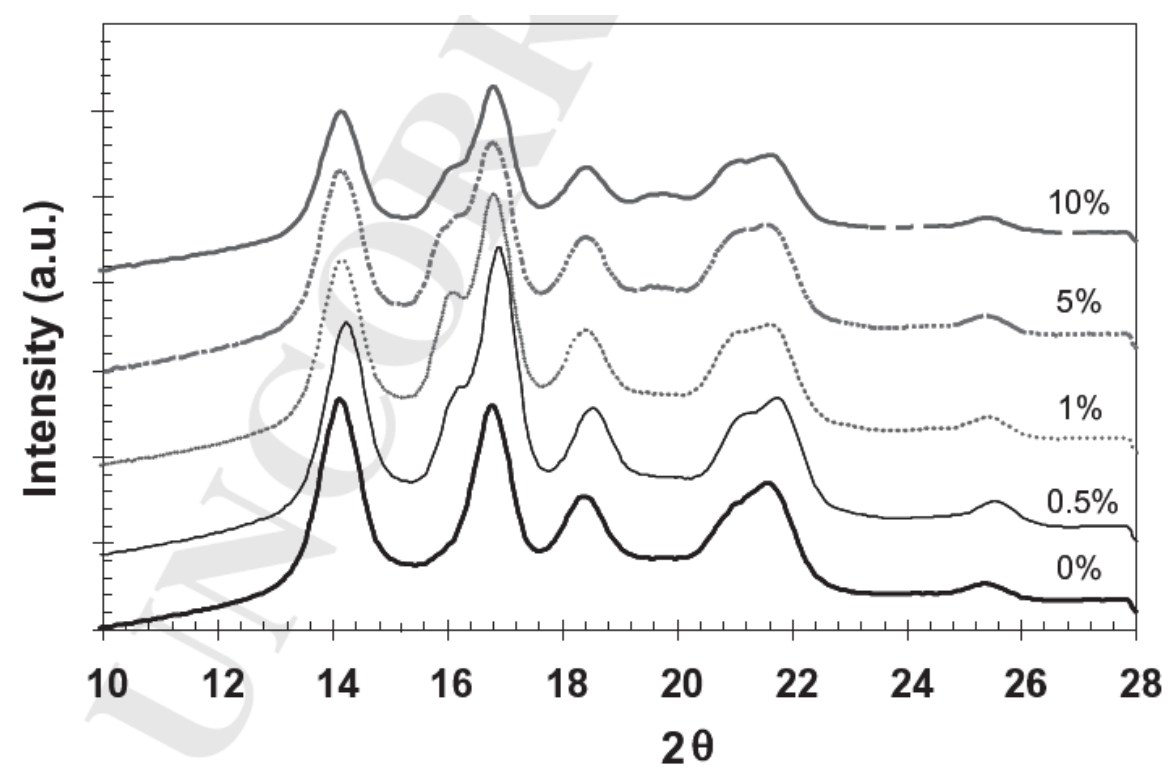

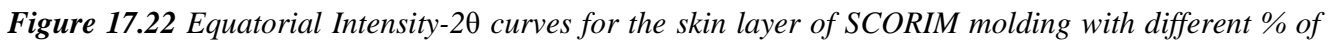
incorporation of MMT (the WAXS patterns are those of Figure 17.21).. 
The use of in situ structure-sensitive experimental techniques during deformation studies are therefore of paramount relevance. Following this approach, small angle $\mathrm{X}$-ray scattering (SAXS) investigations under synchrotron sources may give fundamental insights about deformation mechanisms at the nanoscale with adequate time-resolution.

\subsubsection{Materials and Sample Preparation}

Different organo-modified layered silicate/montmorillonite were used: Nanofil_R 2 and 32 (referenced as NF2 and NF32, respectively) from Sud-Chemie. These nanoclays are functionalized with long chain hydrocarbon/ benzyl groups. NF2 and N3F2 have average initial particle size of 8 and $30 \mu \mathrm{m}$, respectively (agglomerate dimensions), which are composed of platelets with typical dimensions of 100-500 $\mathrm{nm} \times 1 \mathrm{~nm}$ (nanoclay).

The PET nanocomposites with $0.3 \%$ of nanoparticles (and also neat PET for comparision purposes) were prepared via mechanical blending in a tumbler mixer and subsequent melt blending in a special asymmetric mini mixer [64]. Compression molded plates were then prepared and rectangular tensile specimens were cut from them in a hydraulic press.

\subsubsection{Experimental Techniques}

A miniaturized uniaxial stretching machine was positioned perpendicular to the incident X-ray beam to perform tensile tests. Tensile specimens were stretched in situ at a constant velocity ( 5 $\mathrm{mm} / \mathrm{min}$ ) at HASYLAB, A2 soft condensed matter beam line, DESY, Hamburg, Germany, while acquiring two-dimensional SAXS patterns ( $30 \mathrm{~s}$ of accumulation time)

\subsubsection{Results and Discussion}

Figure 17.23 (top) shows the stress-strain curves of the PET nanocomposites, evidencing the different reinforcing nature of the nanoparticles, mainly in terms of stress and deformation levels. Both nanoclay types reinforced the PET, increasing the stress levels and the deformation capabilities.

Figure 17.23 (bottom) also presents some SAXS patterns during stretching. The incorporation of the nanofillers influences the deformation mechanism of PET. This is dependent upon the initial size of the clays agglomerate ( 8 and $30 \mu \mathrm{m}$ for NF2 and NF32, respectively). For the initial strains, $\varepsilon=0.22$, the SAXS patterns are similar for neat and filled PET. For $\varepsilon=0.58$, the SAXS patterns from neat and filled PET differ: the neat PET shows an intense horizontal streak corresponding to voids highly oriented in SD (between highly stretched fibrils that develop parallel to SD); whereas the PET nanocomposites show a cross pattern with an intense streak in the vertical direction (eventually due to external reflection of the interfaces of the crazes with the polymer) and a less intense streak in the horizontal direction (possibly due to elongated voids between fibril is inside the craze) [66]. It may be suggested that the appearance of crazes is delayed (in terms of strain levels) for the PET nanocomposites. Furthermore, at that strain level the void size is slightly bigger for PET reinforced with NF2. At higher deformations, $\varepsilon \geq 0.95$, and for all material systems, large voids are formed highly oriented in the SD, which increase in size with deformation. For the PET nanocomposites, the generated voids are smaller and with a narrower size distribution as compared with the neat PET. For the PET reinforced with NF32 (higher initial particle size), the formed voids are even smaller and have a narrower size distribution when compared to the NF2 nanoclay. PET-NF32 shows the lowest sustained stress level.

The deformation mechanisms in polymer nanocomposites are still not well understood. The adoption of in situ structure-sensitive experimental techniques (mainly X-ray diffraction experiments) during deformation studies is therefore of paramount relevance for identifying the active deformation mechanisms and for establishing the relationships between the structure and mechanical performance. 

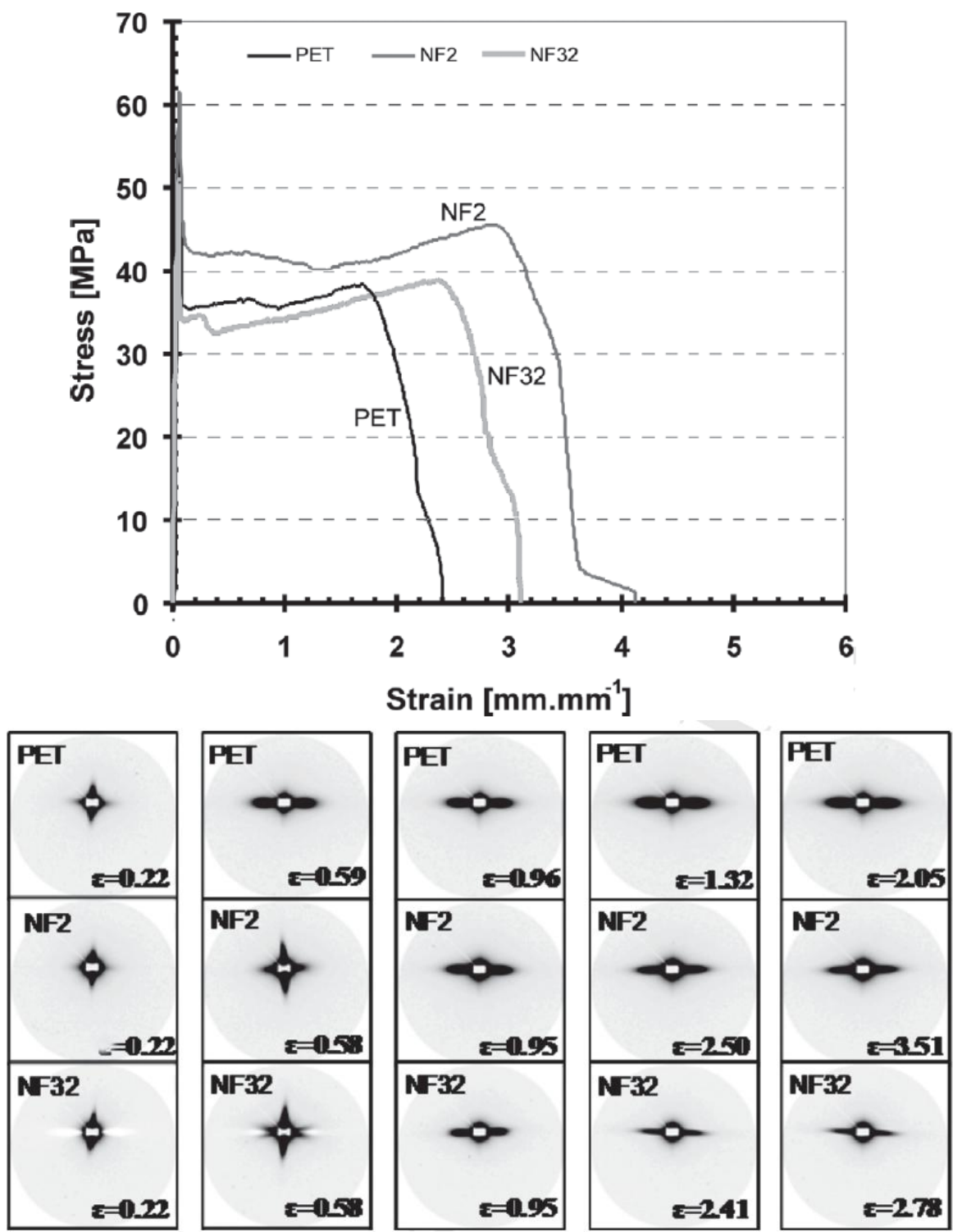

Figure 17.23 Stress-strain curve of PET nanocomposites (top) and correspondent SAXS patterns at different strain levels (bottom) (adapted from [65])

\subsection{Concluding Remarks}

This chapter demonstrates that synchrotron WAXS and SAXS studies can be very useful in studying the relation between the structure and the mechanical properties in multiphasic polymer systems. The standard testing methods and software used for SAXS and WAXS data handling, however, show some limitations and need further development. In most of the instances, the WAXS and SAXS patterns are processed and interpreted, reflection by reflection, so as to extract indirectly the structural information (assuming a structural model). The latter can often be distorted or even damaged due to various reasons related either to the data collection or to the data treatment. The progress in the X-ray experiments during the last years has been tremendous and included development of new two-dimensional X-ray detectors, the use of high power X-ray microbeams, and the application of novel processing methodologies allowing for a direct transformation of the WAXS and SAXS 2D patterns into an image of the 
nanostructure. The calculation of the CDF, briefly discussed in this chapter, can provide structural information absolutely unavailable in other ways. With the advent of the nanotechnologies requiring a strict and rigorous control over the structures on the nanometer scale, this method can be of some importance for both industry and academia. The latest invention is the fast tomographic imaging method based on SAXS data from a scanningmicrobeam experiment [67]. By means of this method, real-time X-ray experiments using mechanical testers for slow or fast load-cycling test can be incorporated into the synchrotron beamline. In such a way, fatigue and failure can be studied in polymer systems within reasonable intervals of time and the data related to microstructure variation inside the material.

\section{Acknowledgements}

This work was supported by DESY and the European Commission under HASYLAB Projects DESY D-II-05-101 EC, DESY-D-II-07-011 EC and the FP6 contract RII3-CT-2004-506008 (IA-SFS). This work was also supported by FCT - Portuguese Foundation for Science and Technology through project POCTI/CTM/46940/2002 (MICROTEST) and POCI/CTM/57358/2004 (NANOFIBCO).

\section{References}

1. L. A. Utracki, in: Preface to Polymer Blends Handbook, Utracki L. A. (Ed.), 2002, Kluwer Academic Publishers, Dordrecht, The Netherlands.

2. L. S. Schadler, Polymer-based and Polymer-filled Nanocomposites in: Nanocomposite Science and Technology, Ajayan P.M., Schadler L.S. and Braun P.V. (Eds), 2003, WileyVCH Verlag GmbH Co. KGaA, Weinheim, p. 77.

3. A. N. Wilkinson, A.J. Ryan, Polymer Processing and Structure Development, 1999, Kluwer Academic Publishers, Dordrecht, the Netherlands.

4. G. Groeninckx and D. Dumpas, Plastic Deformation Mechanisms in Rubber and RubberModified Thermoplastic Polymers: Molecular and Morphological Aspects. In: Structure and Properties of Multiphase Polymeric Materials,

T. Araki, M. Shibayama, Q. Tran-Cong (Eds), 1998, CRC Press, Marcel Dekker Inc, New York, USA, p. 121.

5. D. Schwahn, Critical to Mean Field Crossover in Polymer Blends, Adv. Polym. Sci., 183:163 (2005).

6. K. Mortensen, Small-angle X-ray and Neutron Scattering Studies from Multiphase Polymers, Current Opinion in Solid State \& Materials Science, 2: 653-660 (1997).

7. R. J. Roe, Methods of X-ray and Neutron Scattering in Polymer Science, Oxford University Press Inc., New York, 2000, pp. 90-128.

8. N. Stribeck, X-Ray Scattering of Soft Matter, Springer-Verlag, Berlin, Heidelberg 2007, pp. 42-61.

9. R. J. Davies, C. Riekel, J. A. Bennett, S. J. Eichhorn, R. J. Young, Probing the Internal Geometry of a Woven Composite During Deformation Using an X-ray Microdiffraction Imaging Technique, Appl. Phys. Lett. 91:044102 (2007).

10. C. L. Tucker III, P. Moldenaers, Microstructural evolution in polymer blends, Annual Review of Fluid Mechanics, 34: 177-210 (2002).

11. S. S. Ray, M. Okamoto, Polymer/layered silicate nanocomposites: a review from preparation to processing, Prog. Polym. Sci. 28 (2003) 1539-1641

12. S. C. Tjong, Structural and Mechanical Properties of Polymer Nanocomposites, Materials Science and Engineering R 53 (2006) 73-197

13. M. Evstatiev, S. Fakirov, Microfibrillar Reinforcement of Polymer Blends, Polymer 33:877 (1992)

14. S. Fakirov, M. Evstatiev, J.M. Schultz, Microfibrillar Reinforced Composite from Drawn Polyethylene Terephthalate/Polyamide 6 Blends, Polymer 34, 4669 (1993). 
15. S. Fakirov, M. Evstatiev, S. Petrovich, Microfibrillar Reinforced Composites from Binary and Ternary Blends of Polyesters and Nylon 6, Macromolecules 26:5219 (1993).

16. R. E. Newnham, J.R. Giniewicz, Non-mechanical Properties of Composites, in: Comprehensive Composite Materials, Vol. 1. Fiber Reinforcements and General Theory of Composites, Kelly A. and Zweben C. (Eds), Elsevier, Amsterdam, pp. 431-463 (2000).

17. S. Fakirov, M. Evstatiev, K. Friedrich, Nanostructured Polymer Composites from Polymer Blends: Morphology and

Mechanical Properties, in: Handbook of Thermoplastic Polyesters, Fakirov S. (Ed.), WileyVCH, Weinheim, pp. 1093-1132 (2002).

18. S. Fakirov, M. Evstatiev, K. Friedrich, From Polymer Blends to Microfibrillar Reinforced Composites, in: Polymer Blends, Vol. 11, Formation and Preparation, Paul D.R (Ed.), Bucknall CB, J. Wiley and Sons, Inc., New York, p. 455-476 (2000).

19. A. A. Apostolov, M. Evstatiev, S. Fakirov, A. Kloczkowski, J. E. Mark, Structures and Mechanical Properties of Zone-Drawn-Zone-Annealed Blends of Cocrystallizing Poly(butylene terephthalate) and a Poly(ether ester, J Appl Polym Sci 59:1667 (1996).

20. M. Evstatiev, J. M. Schultz, G. Georgiev, S. Petrovich, S. Fakirov, K. Friedrich, In situ Polymer/Polymer Composites from Poly(ethylene terephthalate), Polyamide-6, and Polyamide-66 Blends, J Appl Polym Sci. 67:723 (1998).

21. D. Sapoundjieva, Z. Denchev, M. Evstatiev, S. Fakirov, N. Stribeck, M. Stamm, Transcrystallization with Reorientation in Drawn PET/PA12 Blend as Revealed by WAXS from Synchrotron Radiation, J Mater Sci 34:3063 (1999).

22. S. Fakirov, M. Evstatiev, Microfibrillar Reinforced Composites - New Materials from Polymer Blends, Adv Mat 6:395 (1994).

23. M. Evstatiev, S. Fakirov, G. Bechtold, K. Friedrich, Structure-Property Relationships of Injection- and Compression-Molded Microfibrillar-Reinforced PET/PA-6 Composites, Adv Polym Techn 19:249 (2000).

24. Z. Denchev, M. J. Oliveira, O. S Carneiro, Nanostructured Composites Based on Polyethylene-Polyamide Blends. I. Preparation and Mechanical Behavior, J Macromol SciPhys, B43:143 (2004).

25. S. Fakirov, H. Kamo, M. Estatiev K. Friedrich, Microfibrillar Reinforced Composites from PET/LDPE Blends: Morphology and Mechanical Properties, J Macromol Sci - Phys B43:775 (2004).

26. K. Friedrich, M. Evstatiev, S. Fakirov, O. Evstatiev, M. Ishii, M. Harras, Microfibrillar Reinforced Composites from PET/PP Blends: Processing, Morphology and Mechanical Properties, Comp Sci Techn 65: 107 (2005).

27. Z. Denchev, N. Dencheva, Transforming Polymer Blends into Composites: A Pathway towards Nanostructured Materials, Polym Int 57:11-22 (2008).

28. A. Monticciolo, P. Cassagnau, A. Michel, Fibrillar Morphology Development of PE/PBT Blends: Rheology and Solvent Permeability, Polym Eng Sci 38: 1882 (1998).

29. I. Pesneau, A. Ait-Kadi, M. Bousmina, P. Cassagnau, A. Michel, In-Situ Polymer-Polymer Composites, ANTEC 99, New York, May 2-6 1999; 2661.

30. M. Evstatiev, S. Fakirov, B. Krasteva, K. Friedrich, J.A. Covas, A.M. Cunha, Recycling of Poly(Ethylene Terephthalate) as Polymer-Polymer Composites, Polym Eng Sci 42:826 (2002).

31. D. Schmidt, D. Shah, E. P. Giannelis, New Advances in Polymer-Layered Silicate Nanocomposites, Current Opinion in Solid State and Materials Science 6, 2002, 205-212.

32. M. Okamoto, Recent Advances in Polymer/Layered Silicate Nanocomposites: An Overview from Science to Technology, Materials Science and Technology, 22(7), 756-779 (2006).

33. C. L Wu, M. Q. Zhang, M. Z. Rong, K. Friedrich, Tensile Performance Improvement of Low Nanoparticles Filled Polypropylene Composites, Compos. Sci. \& Technol., 62:13271240 (2002)

34. H.R. Dennis, D.L. Hunter, D. Chang, S. Kim, J.L. White, J.W. Cho, D.R. Paul, Effect of Melt Processing Conditions on the Extent of Exfoliation in Organoclay-based Nanocomposites, Polymer, 42(23): 9513-9522 (2001).

35. Q. Zhang, Y.Wang, Q. Fu, Shear-induced Change of Exfoliation and. Orientation in Polypropylene/Montmorillonite Nanocomposites, J Polym Sci: Part B: Polym Phy, 41:1-10 (2003).

36. J.J. Hernandez, M.C. Garcia-Gutierrez, A. Nogales, D.R. Rueda, A. Sanz, I. Sics, B.S. Hsiao, Z. Roslaniec, G. Broza, T.A. Ezquerra, Deformation Behaviour during Cold Drawing 
of Nanocomposites Based on Single Wall Carbon Nanotubes and Poly(ether ester) Copolymers, Polymer 48:3286-3293 (2007).

37. L. E. Alexander, X-Ray Diffraction Methods in Polymer Science, Wiley-Interscience, New York, 1969.

38. F. J. Baltá-Calleja, C. G. Vonk, X-ray Scattering of Synthetic Polymers, Elsevier, Amsterdam, 1989.

39. W. Ruland, X-ray Determination of Crystallinity and Diffuse Disorder Scattering, Acta Crystallogr. 14:1180 (1961).

40. Z. W. Wilchinsky, On Crystal Orientation in Polycrystalline Materials, J. Appl. Phys., 30, $792(1959)$

41. C. Santa Cruz, N. Stribeck, H. G. Zachmann, F. J. Balt'a-Calleja, Novel Aspects of the Structure of Poly(Ethylene Terephthalate) as Revealed by means of Small-Angle X-ray Scattering, Macromolecules 24:5980-5990 (1991).

42. G. Kortleve, C. G. Vonk, X-ray Small-Angle Scattering of Bulk Polyehtylene, Kolloid-Z. 225:124-131 (1968).

43. W. Ruland, Evaluation of Small-Angle Scattering of Anisotropic Lamellat Two-Phase Systems by means of Interface Distribution Functions, Colloid Polym Sci 256:932-936 (1978).

44. Z. Denchev Z, T .A. Ezquerra, TA, A. Nogales, I. ` Sics,Microstructural Characterization of Poly(Ethylene Naphthalene 2,6-Dicarbocylate) as Revealed by the Properties of Both Amorphous and Crystalline Phases, in: Handbook of Thermoplastic Polyesters: PET, PBT, PEN - homopolymers, copolymers, blends and composites, S. Fakirov (Ed.), 2002, Wiley$\mathrm{VCH}$, Weinheim, p. 483-546.

45. N. Dencheva, T. Nunes, M. J. Oliveira, Z. Denchev, Crystalline Structure of Polyamide 12 as Revealed by Solid-State 13C NMR and Synchrotron WAXS and SAXS, J. Polym. Sci. Part B: Polym. Phys 43:3720-3733 (2005).

46. N. Dencheva, T. Nunes, M. J. Oliveira, Z. Denchev, Microfibrilar Composites based on Polyamide/Polyethylene Blends; 1. Structure Investigations in Oriented and Isotropic Polyamide 6, Polymer 46:887-901 (2005).

47. V. Causin, C. Marega, A. Marigo, G. Ferrara, Assessing Organo-Clay Dispersion in Polymer Layered Silicate Nanocomposites: A SAXS Approach, Polymer 46:9533-9537 (2005).

48. N. Preschilla, G. Sivalingam, A.S. Abdul Rasheed, S. Tyagi, A. Biswas, J. R. Bellare. Quantification of Organoclay Dispersion and LamellarMorphology in Poly(Propylene)Clay Nanocomposites with Small Angle X-ray Scattering, Polymer 49:4285-4297 (2008).

49. J. Bandyopadhyay, S. S. Ray, The Quantitative Analysis of Nano-clay Dispersion in Polymer Nanocomposites by Small Angle X-ray sScattering combined with Electron Microscopy, Polymer 51:1437-1449 (2010).

50. N. Dencheva, M. J. Oliveira, O. S. Carneiro, A.S. Pouzada, Z. Denchev, Preparation and Structure Development in Microfibrilar Composite Materials based on Polyethylene/Polyamide 6 Oriented Blends, J. Appl. Polym. Sci. 115(5): 2918-2932 (2010).

51. NogalesA,Hsiao BS, Somani RH, Srinivas S, Tsou AH, Balta-Calleja FJ, Ezquerra TA, Shear-induced Crystallization of Isotactic Polypropylene with Different Molecular Weight Distributions: In situ Small- and Wide-angle X-ray Scattering Studies, Polymer 42:5247$5256(2001)$.

52. POLAR software version 2.7.1 is developed by Stonybrook Technology and Applied Research Inc. NY, USA

53. N. Stribeck, S. Fakirov, Three-Dimensional Chord Distribution Function SAXS Analysis of the Strained Domain Structure of a Poly(ether ester) Thermoplastic Elastomer, Macromolecules, 34:7758-7761 (2001).

54. V. Barbi, S. S. Funari, R. Gehrke, N. Scharnagl, N Stribeck, Nanostructure of Nafion Membrane Material as a Function of Mechanical Load Studied by SAXS, Polymer, 44:4853-4861 (2003).

55. Z. Denchev, N. Dencheva, S. S. Funari, M. Motovilin, T. Schubert, N. Stribeck, Nanostructure and Mechanical Properties Studied During Dynamical Straining of Microfibrillar Reinforced HDPE/PA Blends, Journal of Polymer Science: Part B: Polymer Physics, 48(3): 237-250 (2010). 
56. J. P. Nunes, A. S. Pouzada, C.A. Bernardo, The Use of a Three-point Support Flexural Test to Predict the Stiffness of Anisotropic Composite Plates in Bending, Polym Testing 21:2733 (2002).

57. M. van Duin, M. Aussems, R.J.M. Borggreve, Graft Formation and Chain Scission in Blends of Polyamide-6 and-66 with Maleic Anhydride, J. Polym Sci Part A Polym Chem 36:179-188 (1998).

58. Z.-M. Li, B.-H. Xie, S. Yang, R. Huang, M.-B. Yang, Morphology-tensile Behavior Relationship in InjectionMolded Poly(Ethylene Terephthalate)/Polyethylene and Polycarbonate/Polyethylene Blends (II) Part II Tensile Behaviour; J. Mat. Sci., 39:433- 443 (2004).

59. M. Fujiyama, Higher Order Structure of Injection-Moulded Polypropylene, in Polypropylene: Structure, Blends, and Composites, J. Karger-Kcosis (Ed.), Chapman and Hall, London, p.167-204 (1995).

60. S.A. Xu, S.C. Tjong, Deformation Mechanisms and Fracture Toughness of Polystyrene/High-Density Polyethylene Blends Compatibilized by Triblock Copolymer, J. Appl. Polym. Sci., 77, 2024-2033 (2000).

61. G. Kalay, R.A. Sousa, R. L. Reis, A.M. Cunha, M. J. Bevis, The Enhancement of the Mechanical Properties of a High-density Polyethylene, J. Appl. Polym. Sci., 73, 2473-2483 (1999).

62. S. C. Tjong, J.S. Shen and RK.Y. Li, Impact Fracture Toughness of $\beta$-form Polypropylene, Scripta Metallurgica et Materialia, 33(3), 503-508, (1995).

63. S. N. Bhattacharya,M. R. Kamal, R. K. Gupta, in Polymeric Nanocomposites: Theory and Practice, Hanser, Munich, 2008.

64. O. Breuer, U. Sundararaj and R.W. Toogood, The Design and Performance of a New Miniature Mixer for Specialty Polymer Blends and Nanocomposites, Polymer Eng. Sci., 44(5), 868-879 (2004).

65. L. Todorov, A.D. Kouyumdzhiev, D.S. Teixeira, J.C. Viana, S.S. Funari, Evolution of SAXS Patterns during Stretching of PET Nanocomposites, Hasylab Annual Report 2007, part I, 1343-1342 (2007)

66. H. R. Brown, E.J. Kramer, Craze Microstructure from Small-angle X-ray Scattering (SAXS), J. Macromol. Sci., Part B, 19(3), 487-522 (1981).

67. Stribeck N, Nőchel U, Fakirov S, Feldkamp J, Schroer C, Timmann A, Kuhlmann M, SAXS-Fiber Computer- Tomography. Method Enhancement and Analysis of MicrofibrillarReinforced Composite Precursors from PEBA and PET, Macromolecules 41:7637-7647 (2008). 